\title{
Are Forward Designed or Reverse-Engineered UML diagrams more helpful for code maintenance?: A family of experiments
}

\author{
Ana M. Fernández-Sáez ${ }^{\mathrm{a}, *}$, Marcela Genero ${ }^{\mathrm{a}}$, Michel R.V. Chaudron ${ }^{\mathrm{b}}$, Danilo Caivano ${ }^{\mathrm{c}}$, Isabel Ramos ${ }^{\mathrm{d}}$ \\ a ALARCOS Research Group, Instituto de Tecnologías y Sistemas de Información, University of Castilla-La Mancha, Spain \\ ${ }^{\mathrm{b}}$ Joint Computer Science and Engineering Department, Chalmers University of Technology and University of Gothenburg, Sweden \\ ${ }^{\mathrm{c}}$ Department of Informatics, University of Bari, Italy \\ ${ }^{\mathrm{d}}$ Departamento de Lenguajes y Sistemas Informáticos, University of Seville, Spain
}

Software maintenance

UML diagrams

Reverse Engineering

Maintainability Family

of experiments

Controlled experiment
Context: Although various success stories of model-based approaches are reported in literature, there is still a significant resistance to model-based development in many software organizations because the UML is perceived to be expensive and not necessarily cost-effective. It is also important to gather empirical evidence in which context and under which conditions the UML makes or does not make a practical difference.

Objective: Our objective is to provide empirical evidence as to which UML diagrams are more helpful during software maintenance: Forward Designed (FD) UML diagrams or Reverse Engineered (RE) UML diagrams.

Method: We carried out a family of experiments which consisted of one experiment and two replications with a total of 169 Computer Science undergraduate students.

Results: The individual data analysis and the meta-analysis conducted on the whole family, show a tendency in favor of FD diagrams and are significantly different as regards the effectiveness and efficiency of the subjects who participated and played the role of maintainers. The analysis of the qualitative data, collected using a post-experiment survey, reveals that the subjects did not consider RE diagrams helpful. Conclusions: Our findings show that there are some objective results (descriptive statistics or statistical tests) related to the maintenance effectiveness and efficiency in favor of the use of FD UML diagrams during software maintenance. Subjective opinions also lead us to recommend the use of UML diagrams (especially class diagrams) created during the design phase for software maintenance because they improve the understanding of the system in comparison with RE diagrams. Nevertheless, we can only assume that these results are valid in the context of Computer Science undergraduate students when working with small systems related to well-known domains, and other contexts should be explored in order to reaffirm the results in an industrial context by carrying out replications with professionals.

\section{Introduction}

The benefits of using software documentation to comprehend and modify source code have been widely studied [1-3]. The authors of [4] report that having documentation available during system maintenance reduces the time needed to understand how to perform maintenance tasks by approximately $20 \%$.

UML [5] has become the de facto standard modeling notation used to complement software documentation as a graphical

\footnotetext{
* Corresponding author. Tel.: +34 926295300x3740.

E-mail addresses: ana.fernandez@alarcosqualitycenter.com (A.M. Fernández-Sáez), Marcela.Genero@uclm.es (M. Genero), chaudron@chalmers.se (M.R.V. Chaudron), caivano@di.uniba.it (D. Caivano), iramos@us.es (I. Ramos).
}

notation. It first appeared in 1997 and has now become one of the most widely-used modeling languages in industry. There is empirical evidence of its benefits during software development because it increases the understanding between customer and developer and improves communication among team members [6]. UML also improves the source code quality by reducing its defect density [7].

There is also some evidence of the UML's benefits during software maintenance. The availability of UML documentation may result in significant improvements to the functional correctness of changes in addition to their design quality. However, it does not appear that any time is saved as a result. For simpler tasks, the time needed to update the UML documentation may be substantial in comparison with the potential benefits, thus motivating 
the need for UML tools with better support for software maintenance $[8,9]$.

Despite these benefits, there are some common modeling problems that lead to models being less effective and less adopted [10]. For example, some of the limitations of program understating and maintenance supported by UML are the following:

- Unclear specifications of syntax and semantics in some of the UML's more advanced features [3], leading to the need for adequate training in UML [11].

- Spatial layout problems, e.g., large diagrams are not easy to read [3].

- The UML's insufficient support as regards representing the domain knowledge required to understand a program [3].

- The time spent updating the UML diagrams according to the changes in source code counteracts the improvement in source code maintenance time $[8,9]$.

- UML models produced in the requirements analysis process influence neither the comprehensibility of source code nor its modifiability [12].

Despite its limitations, there are clear benefits of using UML, such as the improvement in the traceability from functional requirements to code [11], and the fact that UML is always beneficial in terms of functional correctness (reducing defect density) [13].

Moreover, diagrams with a high level of detail are reported to be more helpful during software development [14], while those with a low level of detail seems to be better when performing maintenance tasks [15].

As we can see, there is some evidence to support the use of UML modeling during software maintenance, but the results are not conclusive owing to the diversity of the results obtained in different empirical studies. It is not clear whether an investment in creating and updating UML diagrams has a return during later phases, like software maintenance which consumes a high percentage of a project's resources. If the presence of UML diagrams is a clear positive factor, but it is not clear what the best way to create them is, then why not create them directly from source code and save the time needed for their creation and update? This is related to a possible influential factor in the software maintenance which has not being studied before: the Origin of the diagrams. FD diagrams, i.e., the diagrams generated during forward development, are sometimes available for maintainers in the maintenance phase, but when this is not the case, the diagrams may be reconstructed through an RE technique [16]. The difference in the origin of the diagrams (i.e., FD diagrams or RE diagrams) and the different techniques that can be used to generate an RE diagram result in different styles of diagrams that may influence the quality of the source code being maintained.

RE diagrams are easier to obtain than FD diagrams because they can be automatically generated with a reversing tool without high investments in developer effort (although these kinds of diagrams need some polishing). Given the ease of their generation and that they can be generated automatically at any time, maintainers can have up-to-date diagrams that model the system when they need them. However, the problem with these diagrams is their very high level of detail, which may lead to difficulties in understanding them. There are several issues related to the obtaining of diagrams with a high level of detail when they originate from source code, after applying a Reverse Engineering technique:

- The level of abstraction is very low, owing to the fact that every element in the source code is represented in the UML diagrams. The benefit of this is that there is a very high traceability from the diagrams to the source code.
- The business rules allow the designers to create UML diagrams by following a specific design objective. The developers then implement the source code by following these diagrams. RE diagrams do not represent these rules, since that they are obtained from source code and these diagrams only reflect how the code was implemented, rather than why.

- These RE diagrams, unlike FD diagrams, are platform-dependent and therefore contain details about the implementation patterns and frameworks used, which do not appear in FD diagrams.

- After obtaining the RE diagrams, a cleaning and lay-outing process needs to be performed in order to adapt them to their corresponding audience.

However, there is another option when up-to-date diagrams are required: the maintainer may keep the source code and the diagrams in-synch manually by applying the corresponding changes incurred by maintenance to both. This option requires more manual effort than the RE process, because the process is not as automated as the RE approach is. Nevertheless, when the diagrams are generated by people and not by automated tools, they may contain different levels of abstraction and detail, depending on the importance of diagram elements [17]; this may make diagrams more understandable, and hence more effective.

All of the above lead us to pose our main research question: "Should software maintenance companies spend time updating their UML diagrams or should they use Reverse Engineered diagrams instead?" Our results might be useful for companies that are performing software maintenance and yet are unsure whether they should continue updating their UML diagrams (as part of the project documentation) or whether they might save that time by automatically generating RE diagrams.

On the one hand, if we obtain better results with design UML diagrams we will have empirical evidence to encourage companies and software developers to follow a model-centric approach. This implies beginning the development of a software system by building the corresponding UML diagrams and keeping them upto-date, thereby facilitating maintenance tasks. On the other hand, if we obtain better results with RE diagrams, we will have empirical evidence to suggest that maintainers should obtain the UML diagrams needed by using RE techniques. This will thus avoid the need to maintain the available diagrams (whenever these are available) and will reduce the time involved in maintenance tasks.

There is a need for experiments focused on software maintenance and evolution by a non-original developer, as this consumes the majority of the resources in a typical software organization.

All of the above led us to perform a family of experiments to investigate whether the different Origins of UML diagrams (Reverse Engineered or Forward Design diagrams) affect the maintainer's performance when modifying source code. Our aim is to discover whether or not, in order to obtain an up-to-date version of diagrams, an effort should be made to maintain diagrams. The family consists of a controlled experiment, which was previously presented in [18], and two replications, carried out with students from two countries (Spain, and Italy). The participants were 169 Computer Science undergraduate students with different abilities and levels of experience with the UML and with JAVA source code.

The three main goals of this paper are therefore to present a thorough description of this family of experiments, its main findings and their practical implications.

This paper is organized as follows. Section 2 presents the related work. Section 3 provides a description of the experiment and replications. The results obtained in the experiment are set out in Section 4, whilst the implications of the study are summarized in Section 5 and the threats to validity are summarized in Section 6. Finally, Section 7 outlines our main conclusions and future work. 


\section{Related work}

The related work will be focused on the empirical evidence on the use of UML diagrams in software maintenance. A Systematic Literature Review that was carried out to collect all the empirical studies performed as regards the use of UML in maintenance and the understandability of UML diagrams which may influence the maintenance of the system is presented in [19]. In this SLR, 46 primary studies were found reporting 74 empirical studies related to this topic, but only the following works directly related to the use of UML diagrams in source code maintenance:

- In [9] an experiment was performed to investigate whether the use of UML influences maintenance in comparison to the use of only source code. This experiment investigates the costs of maintaining and the benefits of using UML documentation during the maintenance and evolution of a real nontrivial system, using 20 professional developers as subjects. These maintainers had to perform 5 maintenance tasks consisting of adding new functionalities to the existing system after which the correctness, time and quality of the solution were measured. Both source code and UML diagrams, when available, had to be maintained. The results of this work show a positive influence of the presence of UML for maintainers. In terms of time, the UML subjects took more time if the UML documentation had to be updated but that difference was not statistically significant. However, UML was always beneficial in terms of functional correctness (introducing fewer faults into the software) because the subjects in the UML group had, on average, a practically and statistically significant $54 \%$ increase in the functional correctness of changes. UML also helped produce code of a better quality when the developers were not yet familiar with the system. This experiment is a replication of a previous work performed with students which is presented in [8] and which obtained similar results. The main difference between [9] and the work presented here is that [9] shows how source code is maintained when UML diagrams are complementing the source code or when the source code is alone, while the subjects of the family of experiments presented herein always received UML diagrams (some received FD diagrams while others received RE diagrams). Another difference is that the UML diagrams used in our family of experiments did not need to be updated according to source code changes. In addition, the subjects of [9] were professionals.

- Arisholm et al. [8] presented the results of two controlled experiments carried out to assess the impact of UML design diagrams on software maintenance. 98 undergraduate students were involved. The authors analyzed the time taken to perform the modifications to the system, the time spent on maintaining the models, and the quality of the modifications performed. The results of the quantitative analysis revealed no significant difference in the time spent making the modifications. Similarly to [9], they observed that the quality of the modifications was higher for those participants who were furnished with UML diagrams. As in [9], the participants' ability and experience were not analyzed with regard to the comprehensibility and modifiability of source code. Unlike our study, the authors analyzed the effect of UML based documentation (a use case diagram, sequence diagrams for each use case, and a class diagram) on modification tasks performed on both UML diagrams and source code. The differences with the work presented in [8] and our work are similar to those mentioned in the case of [9], except for the type of subjects involved.
It is important to mention some other important papers relating to the influence of the use of UML diagrams during software maintenance which were not found as part of [19] because of their dates of publication or because of the objective of the SLR:

- Scanniello et al. [12] used a family of controlled experiments to discover that the use of analysis UML diagrams (those obtained in an early phase or the development process, such as the requirements elicitation or analysis phase) does not significantly improve the comprehension and modifiability of source code with regard to the use of source code alone. These results are valid in the context of undergraduate students and small size systems related to well-known domains. The study presented herein is, however, focused on FD and RE diagrams, which have a higher level of detail. Moreover, the dependent variables are different because the work of Scanniello et al. focused on the comprehension and maintainability of the source code, while the work presented here is focused solely on the maintainability of source code, and bigger systems are also considered.

- In [20] the results of an experiment to assess whether the comprehension of source code is affected when it is added to UML class and sequence diagrams produced in the design phase is presented. The results reveal that the participants benefited from the use of the UML diagrams. An average improvement of $14 \%$ was achieved when the participants accomplished the comprehension task with the class and sequence diagrams, but the time needed to comprehend the code was not significantly influenced from a statistical point of view. Our work differs from that presented in [20] because we compare FD diagrams with RE diagrams rather than with no UML diagrams, and the dependent variable is also different because [20] focuses on the comprehension of the source code and the work presented here is focused on the maintainability of source code. Bigger systems are also used here.

- In the work presented in [21], the experiment performed is focused on the comprehension and the difficulties involved in maintaining object-oriented systems. During this experiment the subjects, who were 34 students in their third year of a Computer Science degree, had to perform 3 different maintenance tasks on a medium-size object-oriented system written in Java. The tasks were related to extending, updating and deleting functionalities of the system, i.e., maintaining the system. It is important to note that the subjects had to give higher priority to the quality of the solutions than to a shorter development time, which may have influenced the results of the experiment. UML diagrams were also presented to the subjects of the experiment, and the correctness of their solutions was measured, but this work was only focused on exploring the participants' strategies and problems while they were conducting maintenance tasks on an object-oriented application. Two major groups of difficulties were related to the comprehension of the application structure, namely the understanding of GUI implementation and 00 comprehension and programming. The main difference between the work presented in [21] and the work here is that the subjects' performance was not measured in the former while it is measured in the latter. In [21] the results are qualitative (description of problems while maintaining source code), while here they are quantitative (results on performance, such as time spent and correctness of the answer).

- The authors of [22] show the results of an explorative survey used to investigate the state of the practice regarding the use of UML in software development and maintenance. The majority of the companies interviewed use UML for software 
development and to perform maintenance operations. Maintenance operations are mainly performed by practitioners with little experience. Another interesting point concerns the average effort needed to perform maintenance operations, which ranges from 1 to 5 person hours for an ordinary maintenance operation (e.g., corrective changes), and from 10 to 50 person hours in the case of an extraordinary maintenance operation (e.g., perfective or adaptive changes). The differences between the work presented in [22] and ours are based on the nature of the empirical study (an explorative survey vs. an explicative family of experiments).

- An experiment presented in [15] studies whether different Levels of Detail (LoD) in UML diagrams might influence the maintenance of source code. In $[14,15]$ there is an assumption that the higher the amount of information put into a diagram, the more is known about the concepts/knowledge described in it. That being the case, a higher LoD would improve maintainers' performances owing to the fact that they would understand the system they have to maintain better. The results from [15] are not conclusive, but show a slight tendency in favor of high LoD diagrams. The similarities of the work presented in this paper with [15] arise from the fact that FD diagrams could be considered as having a high LoD, while RE diagrams could be considered as having a very high LoD. The difference between [15] and the work presented here are the independent variables used (LoD of UML diagrams in the former, and the origin of UML diagrams in the latter), and the design of the experiment (within-subjects and betweensubjects respectively).

Having assumed that the presence of UML diagrams is a positive factor for software maintenance, it is important to know what kinds of UML diagrams are better at improving these kinds of tasks. It is therefore important to additionally study the comprehension of UML diagram in themselves. It is possible to find many papers related to the comprehension of UML diagrams (which is directly related to the comprehension of the software system) in literature [23], [24]. We can highlight two papers found as part of [18], which focus solely on software development using different kinds of UML diagrams during this phase but which might also be related to maintenance:

- The study presented in [14] consists of an empirical experiment focused on determining how different Levels of Detail (LoD) influence the understandability of UML diagrams. It measures the correctness and efficiency in comprehending UML diagrams of 53 Computer Science Master's students. The results show a better understanding of diagrams when they have a high LoD. There are some differences between the work presented in [14] and the family of experiments summarized in this paper. On the one hand, the experiment presented in [14] focuses solely on the comprehension of UML diagrams, and the subjects were not given the source code of the system. The experiment presented in [14] is similar to that presented in [15], but focuses on the development of software rather than the maintenance of software and with the difference that [14] is focused on the comprehension of the UML diagrams and [15] on the maintenance of source code.

- In [3] an experiment with 15 subjects (PhD students or Professors) to assess the qualitative efficacy of UML diagrams in aiding program understanding is described. The experiment had participants analyze a series of UML diagrams and answer a detailed questionnaire concerning a hypothetical software system. Results from the experiment suggest that the relation between the correctness of the solution and the time spent obtaining it using UML to support program understanding is limited by factors such as ill-defined syntax and semantics, spatial layout, and domain knowledge.

All related work that is relevant to this paper is summarized in Table 1 to provide the reader with a better extract of the main information in order to compare the empirical studies. The columns of the table are described as follows:

- Ref: contains the reference to the paper that presents the empirical study considered.

- Type of empirical study: indicates the type of empirical study summarized in the paper (a survey, an experiment, a family of experiments, etc.)

- Goal: describes the goal pursued by the empirical study.

- Subjects: presents the numbers of subjects who participated in the empirical studies and the type of subjects (students, professionals, academic staff, etc.).

- Independent variables: describes the variables that are studied to ascertain their effect on the dependent variables. The values (treatments) of the independent variables are also presented.

- Dependent variables: presents the outcome variables, which are the variables that are affected by the changes produced in the independent variables.

- Experiment design: contains the type of design selected, which can be between-subjects (each subject receives only one treatment) or within subjects (each subject receives all the treatments).

- Tasks: describes the tasks to be performed by the subjects as part of the empirical study.

- Results: reveals the main findings obtained.

It is clear that the LoD of UML diagrams is an important factor which has previously been studied in literature because the LoD of UML diagrams influences their understanding (and hence also source code understanding), and adding details to a diagram is a time consuming task. On the one hand, low LoD diagrams, like analysis models, do not appear to be helpful for source code maintenance [12]. On the other hand, high LoD diagrams are better understood than low LoD diagrams when performing software development [14]. It is therefore clear that a balanced LoD will be the best option, but there is a tendency to use diagrams with a detailed designed, especially when FD are used [20]. But how many details should be presented in a UML diagram? If a high LoD diagram is the best option, why not use an RE diagram, which can be automatically generated (saving time) in comparison to creating UML diagrams during software development and keeping them up-to-date? These questions motivated us to plan a family of experiments that compare the usefulness of FD and RE diagrams when performing source code maintenance.

\section{Description of experiment}

Families of experiments allow researchers to answer questions that are beyond the scope of individual experiments and permit the generalization of findings across studies, thus providing evidence with which to for confirm or reject specific hypotheses [25]. Replications of empirical studies might be regarded as an essential activity in the construction of knowledge in any empirical science based on the following propositions: "We do not take even our own observations quite seriously, or accept them as scientific observations, until we have repeated and tested them" [26] and 
Table 1

Summary of the related work.

\begin{tabular}{|c|c|c|c|c|c|c|c|c|}
\hline Refs. & $\begin{array}{l}\text { Type of } \\
\text { empirical } \\
\text { study }\end{array}$ & Goal & Subjects & $\begin{array}{l}\text { Independent } \\
\text { variables }\end{array}$ & $\begin{array}{l}\text { Dependent } \\
\text { variables }\end{array}$ & $\begin{array}{l}\text { Experiment } \\
\text { design }\end{array}$ & Tasks & Results \\
\hline [9] & 1 Experiment & $\begin{array}{l}\text { Investigates } \\
\text { whether the use } \\
\text { of UML influences } \\
\text { maintenance in } \\
\text { comparison to the } \\
\text { use of only source } \\
\text { code }\end{array}$ & $\begin{array}{l}20 \text { Professional } \\
\text { developers }\end{array}$ & $\begin{array}{l}\text { The use of UML } \\
\text { documentation in a } \\
\text { UML-supported } \\
\text { IDE (possible } \\
\text { values: presence or } \\
\text { absence of UML } \\
\text { diagrams } \\
\text { accompanying } \\
\text { source code) }\end{array}$ & $\begin{array}{l}\text { Time spent } \\
\text { modifying source } \\
\text { code. } \\
\text { Time spent } \\
\text { modifying source } \\
\text { code + UML } \\
\text { diagrams. } \\
\text { Functional } \\
\text { correctness and } \\
\text { quality of the } \\
\text { solution. }\end{array}$ & Between-subjects & $\begin{array}{l}\text { Modify source code } \\
\text { and UML diagrams }\end{array}$ & $\begin{array}{l}\text { The subjects who } \\
\text { received UML } \\
\text { diagrams needed } \\
\text { more time if the UML } \\
\text { documentation was } \\
\text { to be updated. } \\
\text { UML was always } \\
\text { beneficial in terms of } \\
\text { functional } \\
\text { correctness. } \\
\text { UML also helped } \\
\text { produce code of a } \\
\text { better quality when } \\
\text { the developers were } \\
\text { not yet familiar with } \\
\text { the system. }\end{array}$ \\
\hline [8] & 2 Experiments & $\begin{array}{l}\text { Investigates } \\
\text { whether the use } \\
\text { of UML influences } \\
\text { maintenance in } \\
\text { comparison to the } \\
\text { use of only source } \\
\text { code }\end{array}$ & $\begin{array}{l}\text { Undergraduate } \\
\text { students ( } 22 \text { and } \\
76, \text { respectively) }\end{array}$ & $\begin{array}{l}\text { The use of UML } \\
\text { documentation in } \\
\text { a UML-supported } \\
\text { IDE(possible } \\
\text { values: presence } \\
\text { or absence of } \\
\text { UML diagrams } \\
\text { accompanying } \\
\text { source code) }\end{array}$ & $\begin{array}{l}\text { Time needed to } \\
\text { change source } \\
\text { code. } \\
\text { Time needed to } \\
\text { change source } \\
\text { code + UML } \\
\text { diagrams. } \\
\text { Correctness of the } \\
\text { change. } \\
\text { Quality of the } \\
\text { change. }\end{array}$ & Between-subjects & $\begin{array}{l}\text { Modify source code } \\
\text { and UML diagrams }\end{array}$ & $\begin{array}{l}\text { The subjects who } \\
\text { received UML } \\
\text { diagrams needed } \\
\text { more time if the UML } \\
\text { documentation was } \\
\text { to be updated. } \\
\text { UML was always } \\
\text { beneficial in terms of } \\
\text { functional } \\
\text { correctness. } \\
\text { UML also helped } \\
\text { produce code of a } \\
\text { better quality when } \\
\text { the developers were } \\
\text { not yet familiar with } \\
\text { the system. }\end{array}$ \\
\hline [12] & $\begin{array}{l}\text { Family of } \\
\text { experiments } \\
(1+3)\end{array}$ & $\begin{array}{l}\text { Investigates } \\
\text { whether the use } \\
\text { of UML models } \\
\text { produced in the } \\
\text { requirements } \\
\text { analysis process } \\
\text { helps in the } \\
\text { comprehensibility } \\
\text { and modifiability } \\
\text { of source code }\end{array}$ & $\begin{array}{l}\text { Undergraduate } \\
\text { students }(24,22 \text {, } \\
22 \text { and } 18)\end{array}$ & $\begin{array}{l}\text { The use of UML } \\
\text { analysis } \\
\text { models(possible } \\
\text { values: presence or } \\
\text { absence of UML } \\
\text { diagrams } \\
\text { accompanying } \\
\text { source code) }\end{array}$ & $\begin{array}{l}\text { Comprehension of } \\
\text { level of the source } \\
\text { code. } \\
\text { Capability of a } \\
\text { maintainer to } \\
\text { modify source } \\
\text { code. }\end{array}$ & Within-subjects & $\begin{array}{l}\text { Comprehension and } \\
\text { modification tasks. } \\
\text { Post-experiment } \\
\text { questions }\end{array}$ & $\begin{array}{l}\text { UML models } \\
\text { produced in the } \\
\text { requirements } \\
\text { analysis process } \\
\text { influence neither the } \\
\text { comprehensibility of } \\
\text { source code nor its } \\
\text { modifiability }\end{array}$ \\
\hline [20] & 1 Experiment & $\begin{array}{l}\text { Investigates } \\
\text { whether the } \\
\text { comprehension of } \\
\text { source code } \\
\text { increases when } \\
\text { participants are } \\
\text { provided with } \\
\text { UML class and } \\
\text { sequence } \\
\text { diagrams } \\
\text { produced in the } \\
\text { software design } \\
\text { phase }\end{array}$ & $\begin{array}{l}16 \text { Undergraduate } \\
\text { students }\end{array}$ & $\begin{array}{l}\text { The use of } \\
\text { sequence and class } \\
\text { diagrams created } \\
\text { in the design } \\
\text { phase(possible } \\
\text { values: presence or } \\
\text { absence of UML } \\
\text { diagrams } \\
\text { accompanying } \\
\text { source code) }\end{array}$ & $\begin{array}{l}\text { Comprehension of } \\
\text { source code }\end{array}$ & Within-subjects & $\begin{array}{l}\text { Comprehension } \\
\text { questions }\end{array}$ & $\begin{array}{l}\text { Participants } \\
\text { comprehend source } \\
\text { code significantly } \\
\text { better when it is } \\
\text { added to class and } \\
\text { sequence diagrams } \\
\text { together }\end{array}$ \\
\hline [21] & 1 Experiment & $\begin{array}{l}\text { Investigates the } \\
\text { comprehension } \\
\text { and the } \\
\text { difficulties } \\
\text { involved in } \\
\text { maintaining } \\
\text { object-oriented } \\
\text { systems }\end{array}$ & $\begin{array}{l}34 \text { Undergraduate } \\
\text { students }\end{array}$ & - & $\begin{array}{l}\text { Comprehension } \\
\text { and modification } \\
\text { of systems }\end{array}$ & Between-subjects & Modification questions & $\begin{array}{l}\text { Detection of common } \\
\text { difficulties in } \\
\text { understanding when } \\
\text { maintaining software } \\
\text { systems }\end{array}$ \\
\hline [22] & 1 Survey & $\begin{array}{l}\text { Investigates the } \\
\text { state of the } \\
\text { practice regarding } \\
\text { the use of UML in } \\
\text { software } \\
\text { development and } \\
\text { maintenance in }\end{array}$ & - & - & - & - & - & $\begin{array}{l}\text { The majority of the } \\
\text { companies } \\
\text { interviewed use UML } \\
\text { for software } \\
\text { development and to } \\
\text { perform } \\
\text { maintenance }\end{array}$ \\
\hline
\end{tabular}


Table 1 (continued)

\begin{tabular}{|c|c|c|c|c|c|c|c|c|}
\hline Refs. & $\begin{array}{l}\text { Type of } \\
\text { empirical } \\
\text { study }\end{array}$ & Goal & Subjects & $\begin{array}{l}\text { Independent } \\
\text { variables }\end{array}$ & $\begin{array}{l}\text { Dependent } \\
\text { variables }\end{array}$ & $\begin{array}{l}\text { Experiment } \\
\text { design }\end{array}$ & Tasks & Results \\
\hline [15] & 1 Experiment & $\begin{array}{l}\text { Determines the } \\
\text { influence of } \\
\text { different levels of } \\
\text { detail (LoD) of } \\
\text { UML diagrams in } \\
\text { source code } \\
\text { maintenance }\end{array}$ & $\begin{array}{l}11 \text { Undergraduate } \\
\text { students }\end{array}$ & $\begin{array}{l}\text { The level of detail } \\
\text { of UML diagrams } \\
\text { (possible values: } \\
\text { high or low LoD) }\end{array}$ & $\begin{array}{l}\text { Understandability } \\
\text { and modifiability } \\
\text { of source code }\end{array}$ & Between-subjects & $\begin{array}{l}\text { Comprehension } \\
\text { tasks + modification } \\
\text { tasks + subjective } \\
\text { questions }\end{array}$ & $\begin{array}{l}\text { operations. } \\
\text { Maintenance } \\
\text { operations are } \\
\text { mainly performed by } \\
\text { practitioners with } \\
\text { little experience. } \\
\text { No significant results } \\
\text { in favor of high or } \\
\text { low LoD. There is a } \\
\text { slight tendency in } \\
\text { favor of low LoD } \\
\text { diagrams }\end{array}$ \\
\hline [14] & 1 Experiment & $\begin{array}{l}\text { Determines the } \\
\text { influence of } \\
\text { different levels of } \\
\text { detail (LoD) on } \\
\text { the } \\
\text { understandability } \\
\text { of UML diagrams }\end{array}$ & $\begin{array}{l}54 \text { Master's } \\
\text { students }\end{array}$ & $\begin{array}{l}\text { The level of detail } \\
\text { of UML diagrams } \\
\text { (possible values: } \\
\text { high or low LoD) }\end{array}$ & $\begin{array}{l}\text { Comprehension of } \\
\text { UML diagrams }\end{array}$ & Between-subjects & $\begin{array}{l}\text { Comprehension } \\
\text { tasks }\end{array}$ & $\begin{array}{l}\text { Results show that the } \\
\text { effect of LoD in UML } \\
\text { models on model } \\
\text { comprehension is } \\
\text { significant in favor of } \\
\text { high LoD }\end{array}$ \\
\hline [3] & 1 Experiment & $\begin{array}{l}\text { Assesses the } \\
\text { qualitative } \\
\text { efficacy of UML } \\
\text { diagrams in } \\
\text { aiding program } \\
\text { understanding }\end{array}$ & $\begin{array}{l}15 \text { Subjects (PhD } \\
\text { students or } \\
\text { Professors) }\end{array}$ & - & $\begin{array}{l}\text { Comprehension of } \\
\text { UML diagrams }\end{array}$ & Between-subjects & $\begin{array}{l}\text { Comprehension } \\
\text { questions + } \\
\text { modification } \\
\text { questions }\end{array}$ & $\begin{array}{l}\text { UML's efficacy in } \\
\text { support of program } \\
\text { understanding is } \\
\text { limited by factors } \\
\text { such as ill-defined } \\
\text { syntax and } \\
\text { semantics, spatial } \\
\text { layout, and domain } \\
\text { knowledge }\end{array}$ \\
\hline
\end{tabular}

“... replication is needed not merely to validate one's findings, but more importantly, to establish the increasing range of radically different conditions under which the findings hold, and the predictable exceptions".

In order to run and report this family of experiments, we followed the recommendations provided in several pieces of work [27-29]. The family of experiments followed the guidelines for reporting empirical research in software engineering [28] as closely as possible. The experimental material is available for downloading at: http://alarcos.esi.uclm.es/originUMLmaintenance/.

In the following subsections we shall describe the main characteristics of the experiment and the replications, including goal, context, variables, subjects, design, hypotheses, material, tasks, experiment procedure and analysis procedure. Fig. 1 presents the chronology of the family of experiments, summarizing the name of the experiment, the number of participants, and the name of the Universities at which the experiment and replications were run.

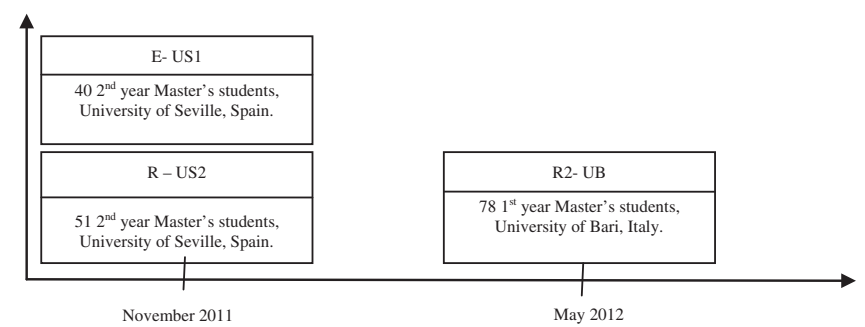

Fig. 1. Chronology of the family of experiments.

\subsection{Goal}

The principal goal of this family of experiments was to investigate whether the Origin of UML diagrams influences the maintenance of source code. The GQM template for goal definition $[25,30]$ was used to define the goal of our experiment as follows: "Analyze the maintainability of source code from the point of view of software maintainers with regard to the Origin of the UML diagrams, in the context of Computer Science students at the Universities of Seville and Bari".

We considered two possible Origins of the diagrams: the design phase and an RE technique. In the first case, our intention was to maintain the source code using the UML diagrams built during the design phase. In the second case, we set out to maintain a source code for which the UML diagrams were not available, which meant that they would have to be obtained from the source code using an RE technique.

We decided to consider class diagrams and sequence diagrams because they can be obtained from an RE technique and because they are also two of the most commonly used diagrams when designing a system $[23,31,32]$.

\subsection{Context selection}

The experimental objects consisted of class and sequence diagrams and the Java code of one system. The diagrams were obtained from different Origins:

- RE: Reverse Engineered UML diagrams, which are constructed completely automatically based on the source code.

- FD: Forward Designed UML diagrams obtained at the design phase. These are totally manually designed. 
Table 2

Metrics of the software system used in the family of experiments.

\begin{tabular}{llllll}
\hline & $\begin{array}{l}\text { \# Class } \\
\text { diagrams }\end{array}$ & \# & $\begin{array}{l}\text { \# Sequence } \\
\text { diagrams }\end{array}$ & $\begin{array}{l}\text { \# } \\
\text { Messages }\end{array}$ & LoC \\
\hline D & 4 & 16 & 21 & 226 & 5123 \\
RE & 4 & 21 & 11 & 191 & \\
\hline
\end{tabular}

RE diagrams are diagrams with a high LoD since they represent all the elements in the source code. The FD diagrams might also be considered as diagrams with a high LoD because their class diagrams contain class names, attributes, operations and relationships, and their sequence diagrams contain lifelines, messages and parameters of messages. However, FD diagrams do not represent all the elements in the source code, but those elements which are represented (based on human selection) are completely represented. FD diagrams can therefore be considered as high LoD diagrams while RE are higher LoD diagrams.

The diagrams described a sports center system from which users can rent services (tennis courts, etc.). The system is a sports center application which was created as part of the Master's degree Thesis of a student from the University of Castilla-La Mancha, and we therefore consider it to be a realistic system. It is a desktop application created with the client-server paradigm. The system contains 5123 Lines of Code (LoC) (Table 2), so it might be considered a small realistic system. In fact its size is almost double the LoC of other systems used in previous works which have nevertheless been considered as realistic systems, for example in [9]. The maintenance requirements were formulated by the Master's supervisor. In the case of the FD diagrams, 4 class diagrams are available, with a total of 16 classes, and 21 sequence diagrams, with 226 messages. In the case of RE diagrams, 4 class diagrams are available, with 21 classes, and 11 sequence diagrams, with 191 messages. The number of classes in class diagrams is a good deal smaller than in the class diagrams of systems used in other previous work. This is owing to the use of different levels of abstraction for modeling, but their diagram size is still representative of realistic systems [33]. Note that the number of sequence diagrams in the RE group is 11 and the number of diagrams in D group is 21 . The number of messages per diagram (226 messages for 21 diagrams in D group, and 191 messages for 11 diagrams in RE group) therefore provides us with an indicator which suggests that RE diagrams should be considered as being larger and more complex. The RE diagrams were generated using the IBM Rational Software Architect tool, employing the default RE-functionality that it provides, followed by auto-lay-outing (also offered by the same tool). These experimental objects were presented in Spanish in E-US1 and R-US2. A native Italian speaker translated all the material into Italian in the case of R-UB. The replicators supported the native speakers and helped them when needed (e.g., in the translation of technical terms).

The participants, who were grouped by experiment, had the following characteristics:

- E-US1: The participants were 40 Computer Science students from the University of Seville (Spain) who were taking the Software Engineering III course in the second-year of their Master's Degree, from which they had acquired training in UML diagrams (as they also had from previous Software Engineering courses). $90 \%$ of the students were non-repeating course students and most of them did not have industrial experience (five subjects had experience as programmers, another as an analyst and another as a maintainer). The subjects considered their knowledge of the UML to be high/very high in most cases for class diagrams, and medium/high for sequence diagrams (the two kinds of diagrams which were used during the experiment). Most of the subjects' knowledge of JAVA language is excellent (all the subjects considered it to be above average). In Spain, grades are expressed as doubles and assume values of between 5 and 10 . The lowest grade is 5 , while the highest is 10 .

- $R$-US2: The participants were 51 students from the University of Seville (Spain) who were taking the same course as the students in the original experiment, i.e., they were taking the Software Engineering III course in the second-year of their Master's Degree, from which they had acquired training in UML diagrams (as they also had from previous Software Engineering courses).In this case, more than half (58\%) the students were repeating the course and most of them had industrial experience, mainly as programmers, but also as analysts, designers or maintainers $(40 \%$ of the subjects did not have any experience at all). The subjects considered their knowledge of the UML to be high/very high in most cases for class diagrams and for sequence diagrams, and most of them had an excellent knowledge of JAVA language (all the subjects considered this to be above average). The grading system is analogous to that explained for E-US1.

- $R$-UB: The participants were 78 Computer Science students who were taking the Software Engineering course in the second-year of their Laurea Degree at the University of Bari. In Italy, the exam grades are expressed as integers and assume values of between 18 and 30 . The lowest grade is 18 , while the highest is 30. We cannot provide details about their background because the first session of the experiment needed to be canceled for reasons beyond our control.

The students who participated in the experiment and the replications were volunteers selected for convenience (the students available in the corresponding course).

\subsection{Variable selection}

The independent variable (also called the "main factor") is the Origin of diagrams, which is a nominal variable with two values (treatments):

- Forward Designed (FD).

- Reverse Engineered (RE).

The dependent variable is the maintainability. This dependent variable was measured using the following measures:

- Maintainability Effectiveness (MEffec): This measure is related to the correctness of the response, and it therefore reflects the ability to maintain the system presented correctly. A higher value of this measure reflects better Maintainability Effectiveness. It is calculated with the following formula:

$\frac{\text { \# correct tasks - \# performed tasks }}{\# \text { tasks }}$

- Maintainability Efficiency (MEffic): This measure is related to the timing of the response, but also reflects the ability to maintain the system presented correctly. Its unit of measure is "the number of correctly-performed modification tasks per time unit". The unit of time used was seconds. A higher value of this measure reflects better maintainability efficiency. It is calculated with the following formula: 
\# correct tasks - \# performed tasks time spent

We also considered a further independent variable (from here on termed as the "cofactor"): Ability. We considered this cofactor in our efforts to investigate whether subjects' abilities play any role in the maintenance of source code, i.e., we discriminate between users according to their respective level of Ability, with the purpose of testing the hypothesis that this is a relevant influencing factor that should be taken into account when adopting these kinds of diagrams. A quantitative assessment of the participants' Ability was obtained by computing the final grade of the course they were taking. Those students from E-US1 and R-US2 with a final grade of below 5.7/10 (which represents the median of the group) were classified as low Ability participants, while those with a higher grade were given the classification of high Ability students. The instructor of the course (the last author of the paper), who was not one of the experimenters, was asked to provide the grades. In the case of R-UB, the cutoff point was 2.5/5.

\subsection{Hypotheses formulation}

The following hypotheses have been formulated and tested:

- $H_{1,0}$ : there is no significant difference in the subjects' maintenance effectiveness when working with UML diagrams which have originated from the design phase or with diagrams which have originated from a Reverse Engineering technique. $\mathrm{H}_{1,1}$ : $\mathrm{H}_{1,0}$.

- $H_{2,0}$ : there is no significant difference in the subjects' maintenance efficiency when working with UML diagrams which have originated from the design phase or with those which have originated from a Reverse Engineering technique. $\mathrm{H}_{2,1}: \mathrm{H}_{2,0}$.

The goal of the statistical analysis is to reject the null hypotheses and possibly to accept the alternative ones. Both of the hypotheses are two-sided, because we did not postulate any effect arising from the origin of the diagrams.

\subsection{Experimental design}

When designing the experiment we attempted to alleviate several issues that might threaten the validity of the research done by considering the suggestions provided in [29].

Table 3

Experimental design.

\begin{tabular}{ll}
\hline Origin of UML diagrams & \\
\hline $\mathrm{RE}$ & $\mathrm{D}$ \\
\hline Group 1 & Group 2 \\
\hline
\end{tabular}

We selected a between-subjects balanced design in which each treatment has an equal number of subjects [34]. We decided to use a between-subjects design rather than a within-subjects design owing to time constraints. The inherent threats of a betweensubjects design were thus alleviated by taking into account the suggestions provided in [29]. In an attempt to alleviate experience effects, we provided the subjects with a background questionnaire (which can be downloaded with the rest of the experiment material) in the training session which took place before carrying out the experiment. The background questionnaire consisted of three blocks of questions. The first one was related to the subjects' skills and experience. The second block consisted of a set of question used to measure the subjects' knowledge of UML. The last block was a set of questions about Java. This background questionnaire improved on the design of this family of experiments in comparison with the previous experimentation work presented in the related work section.

The subjects were then assigned to the 2 groups in a random manner (see Table 3), based on the marks obtained in the background questionnaire (blocked design by experience). This was not possible in the case of the R-UB subjects, who were assigned to each group in a random manner.

To avoid skewing the results of the tasks as a result of their being of different levels of difficulty, they were randomized. The subjects in each group therefore received the same tasks but in a different order. In order to alleviate learning effects, the order of the tasks was the same for each treatment, i.e., one subject from each group received the tasks in the same order, but in a different order from the rest of his/her group.

\subsection{Experimental tasks}

The modification questionnaires were formed of two kinds of maintenance tasks (Table 4); both of these activities involve changing the source code:

- Adaptive maintenance task: these maintenance activities were intended to enhance the system by adding features, capabilities, and functions, in response to new technology, upgrades, new requirements, or new problems, i.e., a modification of a software product performed after delivery to keep a software product usable in a changed or changing environment [35]. In our case, new requirements had to be added to the system, with the subjects receiving a list of requirements which had to be used to modify the code of the system and thus add/change certain functionalities. This part of the experiment consisted of 3 tasks (see example in Fig. 2).

- Corrective maintenance task: these maintenance activities were "intended to remove errors or bugs from the software, the procedures, the hardware, the network, the data structures, and the documentation" [36]. In our case, bugs from the source code had to be detected and fixed. We consequently analyzed the list of bugs reported by a professional Dutch IT development

Table 4

Summary of maintenance tasks.

\begin{tabular}{|c|c|c|c|}
\hline Task & Summary of task descriptions & $\begin{array}{l}\text { Type of } \\
\text { maintenance }\end{array}$ & $\begin{array}{l}\text { Maximum } \\
\text { mark }\end{array}$ \\
\hline T1 & $\begin{array}{l}\text { When one of the sport center's services is not available (owing to a breakdown, for example) all reservations for this service } \\
\text { should be cancelled }\end{array}$ & Corrective & 4 Points \\
\hline $\mathrm{T} 2$ & The sport center's system should store its customers' telephone numbers & Adaptive & 5 Points \\
\hline T3 & A ticket showing a customer's reservations at a specific time should be generated by the system & Adaptive & 5 Points \\
\hline T4 & When we delete one of the sport center's members, his/her pending payments sometimes remain in the system & Corrective & 2 Points \\
\hline T5 & The information about the sport center's instructors should be stored by the system & Adaptive & 6 Points \\
\hline
\end{tabular}




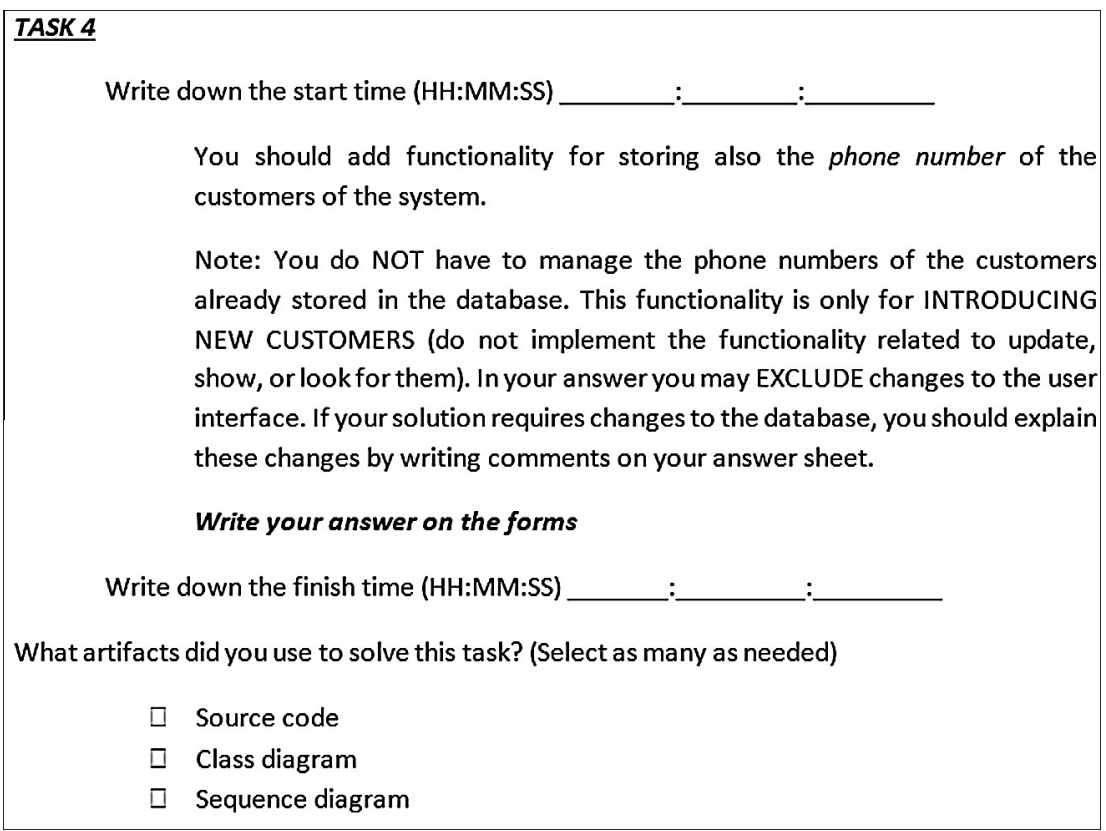

Fig. 2. Example of adaptive maintenance task.

\begin{tabular}{|c|c|}
\hline \multicolumn{2}{|l|}{ TASK 5} \\
\hline \multicolumn{2}{|r|}{ Write down the start time (HH:MM:SS) ___ _ _ _ _ } \\
\hline & $\begin{array}{l}\text { A bug has been detected in the system. When a member of the sport center is } \\
\text { deleted, his/her outstanding payments remain in the system in some cases. } \\
\text { Locate the error and fix it. In your answer you may EXCLUDE changes to the } \\
\text { user interface. If your solution requires changes to the database, you should } \\
\text { explain these changes by writing comments on your answer sheet. }\end{array}$ \\
\hline & Write your answer on the forms \\
\hline Write & lown the finish time (HH:MM:SS) \\
\hline \multicolumn{2}{|c|}{ What artifacts did you use to solve this task? (Select as many as needed) } \\
\hline$\square$ & Source code \\
\hline$\square$ & Class diagram \\
\hline$\square$ & Sequence diagram \\
\hline
\end{tabular}

Fig. 3. Example of corrective maintenance task.

company (whose name is not shown for reasons of privacy) and introduced these kinds of defects into our system, giving the subjects a list of functional defects which had to be detected and corrected. All this explains why we consider these tasks to be common, realistic tasks. This part of the experiment consisted of 2 such tasks (see example in Fig. 3). The subjects were provided with answer sheets for this kind of questions, to allow them to structure their responses.

These two kinds of tasks needed to be answered using data collection forms, i.e., templates which had to be filled in with pieces of code (see example in Fig. 4). We used these data collection forms to obtain a structured response which facilitated the correction of the results, and is also an improvement on the design of this family of experiments in comparison with previous work. The subjects were provided with answer sheets to allow them to structure their responses regarding the maintenance tasks. The reason for doing this was that maintaining source code on paper is not easy owing to space constraints, and the subjects were therefore required to write changes to the source code in a structured manner on the answer sheets (format: line-no, change type, Java code, etc.). They had to fill in a different form depending on the element that they wished to maintain (a class, a method, an attribute, etc.). These answer sheets can be found at: http://alarcos.esi.uclm.es/originUMLmaintenance/.

The largest change consisted of adding a class which would need at least 22 lines of code. In general, between 1 and 3 classes needed to be modified. The complexity of the task might not appear to be too complex, owing to the number of LoCs that have to be changed, but the complexity of the task lies in the 


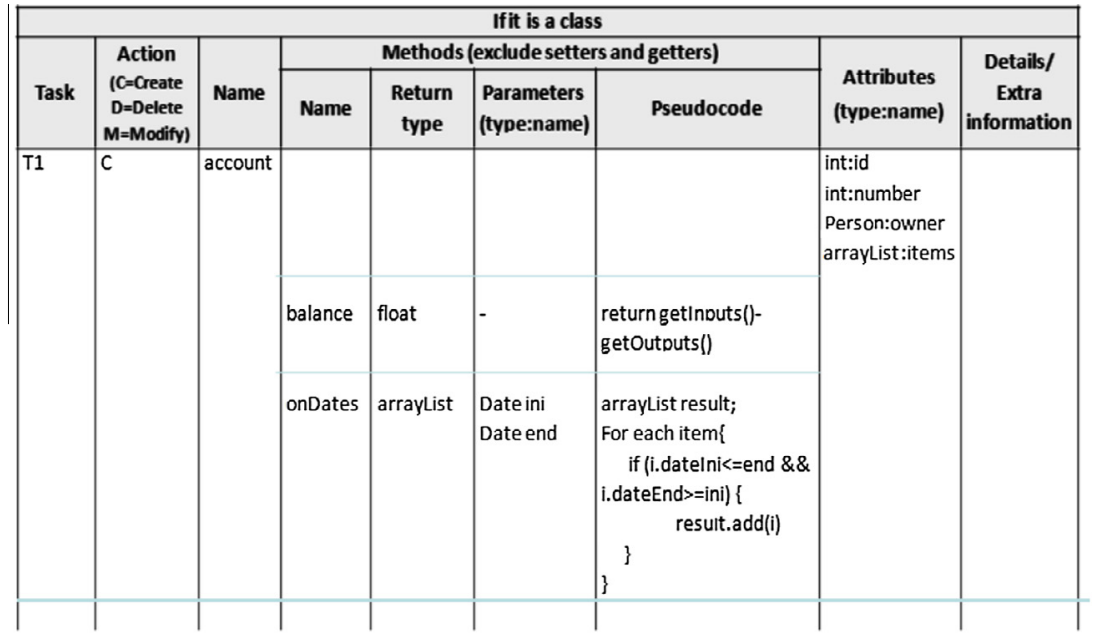

Fig. 4. Answer sheet related to the element "Class", filled with an example.

Table 5

Post-experiment survey.

\begin{tabular}{|c|c|c|}
\hline Id & Question/issue & Possible answers \\
\hline Ex1 & The difficulty of tasks & $(1-5)$ \\
\hline Ex2 & The training was sufficient to be able to perform the tasks & $(1-5)$ \\
\hline Ex3 & The clarity of the material provided & $(1-5)$ \\
\hline Ex4 & The task objectives were perfectly clear to me & $(1-5)$ \\
\hline Ex5 & The tasks I performed were perfectly clear to me & $(1-5)$ \\
\hline Ex6 & I did not experience difficulty in reading the diagrams & $(1-5)$ \\
\hline Ex7 & I did not experience difficulty in reading the source code & $(1-5)$ \\
\hline Ex8 & The LoD of the diagrams was correct enough for me to be able to perform the tasks & $(1-5)$ \\
\hline Ex9 & The available class diagrams were helpful & $(1-5)$ \\
\hline Ex10 & In the event that you do not think that the class diagrams have been useful, indicate why & Open question \\
\hline Ex11 & The available sequence diagrams were helpful & $(1-5)$ \\
\hline Ex12 & In the event that you do not think that the sequence diagrams have been useful, indicate why & Open question \\
\hline Ex13 & I had enough time to perform the tasks & Multiple choice question \\
\hline Ex14 & How much time (as a percentage) did you spend looking at the diagrams? & Multiple choice question \\
\hline Ex15 & How much time (as a percentage) did you spend looking at the source code? & Multiple choice question \\
\hline
\end{tabular}

1 = Strongly agree; 2 = agree; 3 neutral; 4 = disagree; 5 = strongly disagree (Ex2, Ex3, Ex4, Ex5, Ex6, Ex7, Ex9, Ex11).

1 = Very high; 2 = high; 3 = correct; 4 = low; 5 = very low (Ex8).

$1=$ Very difficult; 2 = difficult; $3=$ medium; 4 = easy; 5 = very easy (Ex1).

1 = Very clear; 2 = clear; 3 = correct; 4 = unclear; 5 = very unclear (Ex3).

$\mathrm{A}=$ More time needed; $\mathrm{B}=$ less time needed; $\mathrm{C}=$ enough time (Ex13).

A. $<20 \%$; B. $\geqslant 20 \%$ and $<40 \%$; C. $\geqslant 40 \%$ and $<60 \%$; D. $\geqslant 60 \%$ and $<80 \%$; E. $\geqslant 80 \%(\operatorname{Ex} 14$, Ex 15$)$.

difficulty of detecting where the change is to be made in the source code, and how it should be carried out. It should also be borne in mind that 5 tasks had to be completed in $2 \mathrm{~h}$, using a system that the subjects had never seen. We limited the time of the experiment to fit in with subjects' availability. The subjects were only required to maintain the system, i.e., they did not need to update diagrams according to their changes or to create test cases.

After performing each maintenance task, the subjects were also required to indicate which artifacts (source code, class diagrams and/or sequence diagrams) they had used to solve the task. They were asked this in order to check whether or not they used the diagrams to solve the maintenance tasks (otherwise, the effect measured would not be the influence of the different origin of the diagrams). We shall refer to these kinds of questions as "postquestions".

In addition, at the end of the experiment's execution the subjects were asked to fill in a post-experiment survey (see Table 5), whose goal was to obtain feedback about their perception of the experiment execution - feedback which could be used to explain the results obtained. The answers to the questions were based on a five-point Likert scale [37]. During the experiment's execution, the subjects had to perform 5 maintenance tasks, in different orders, which are summarized in Table 4.

\subsection{Experimental procedure}

The experimental material and the time duration were checked by carrying out a pilot study with $6 \mathrm{PhD}$ students from the University of Castilla-La Mancha, Spain, before the execution of the first experiment. The pilot study was similar to the experiment described in this section, but with no time limit. The results of the pilot study were used as a basis to adapt the number of tasks and their complexity to the experimental time constraints. Some spelling mistakes were also corrected and some requirement statements were rewritten in order to make them more understandable.

We performed internal replications of the original experiment, and the entire family of experiments was carried out by the same experimenters. We conducted the experiment and the replications in a classroom under controlled conditions.

We did not provide details on the experimental hypotheses, and informed the participants that their grade on the course would not be affected by their performance. 
The experiment and replications took place in two sessions of two hours each:

- Training session: The subjects first attended a training session in which detailed instructions on the experiment were presented and the main concepts of UML and JAVA were revised. In this session, we did not provide details on the experimental hypotheses and the subjects carried out an exercise similar to those in the experimental tasks in collaboration with the instructor. During the training session, the subjects were required to fill in a background questionnaire. The participants were informed that the data collected in the experiments were to be used for research purposes and would be treated as confidential, and that their grade on the course they were taking would not be affected by the grade obtained in the experiment. After the introductory lesson, we assigned the participants to one of the 2 groups in accordance with the marks obtained in the background questionnaire, thus obtaining balanced groups. Those subjects who did not come to the training session were randomly assigned to the groups, which in some cases led to unbalanced groups (a difference of 1 subject).

- Execution session: The execution of the experiment took place in the second session, in a classroom, where the students were supervised by the instructor of the course (a different one depending on the replication) and one experimenter (always the same one), and no communication between them was allowed. Each of the groups was given a different treatment.

After the execution of the experiment, the data collected from it were placed on an excel sheet, following an answering diagram constructed before the experiment was carried out. On this sheet, each task has a maximum mark (see Table 4), depending on the correctness of the answer provided. This means that for each task, a mark was given to the subject depending on the number of correct lines of code added to the solution. Incorrect answers were not given negative marks, i.e., lines of code which do not solve the task.

\subsection{Analysis procedure}

The data analysis was carried out by considering the following steps:

1. We first carried out a descriptive study of the measures of the dependent variable, i.e., MEffec and MEffic in order to obtain a general overview of the influence of the main factor (Origin).

2. We then analyzed the characteristics of the data to determine which parametric or non-parametric test it would be better to use. We performed a Kolmogorov-Smirnov test [38] to determine the normality of distributions and a Levene [38] test to determine the homogeneity of variances. These analyses are useful in determining which parametric or non-parametric test it is best to use.

3. Based on the results of the previous test, for the data collected in each experiment we tested the hypotheses formulated using the parametric ANOVA test [39] when the results of Kolmogorov-Smirnov test and Levene test were positive. The non-parametric Mann-Whitney test [38] was performed when the data obtained did not satisfy the restrictions of the ANOVA test (we did not obtain normal distributions, and there is no homogeneity of variances).
4. To strengthen the results of each experiment, we decided to integrate them using a meta-analysis. A meta-analysis is a set of statistical techniques which are used to combine the different effect sizes of the experiments in order to obtain a global effect of a factor in a dependent variable.

5. We analyzed the influence and the interaction of the cofactor, i.e., Ability, using statistical tests. The interaction of the Ability with the main factor (i.e., Origin) was also tested using interaction plots [39]. Interaction plots are simple line graphs in which the means on the values of a dependent variable for each level of one factor are plotted on all the levels of the second factor. The resulting lines are parallel when there is no interaction and nonparallel when an interaction is present.

6. The data collected from the post-experiment survey were eventually analyzed using bar graphs. In cases in which a pattern was detected in the data, these data were also tested with a T-test [38] owing to their nature.

All the statistical values were calculated using SPSS [40], with its standard configuration. In all the statistical tests, we decided to accept a probability of $5 \%$ of committing a Type-I-Error [38].

\subsection{Documentation and communication}

Issues such as documentation [41] and communication among experimenters [42] may influence the success or the failure of an experiment performance and its future replications. Laboratory packages and knowledge-sharing mechanisms were used to handle these issues. The material was originally written in Spanish for E-US1 and R-US2, and was then translated into Italian for R-UB. The material included: the post-experiment survey, the modification questionnaires, the data collection forms, the source code and the UML diagrams (two versions: D and RE). The groups of experimenters also shared a document to provide a common background so as to be able to communicate all terms related to the design and analysis of the experiment.

The experimenters (the first three authors of the paper) began with an initial face-to-face meeting in which the main ideas of the experiments were discussed and reported in an agreement document. All the experimenters then exchanged the agreement documents from the meeting by e-mail to attain a shared common research plan. This phase played a significant role in sharing knowledge among the experimenters and in the discussions on possible issues related to the study that might arise.

The experimenters used instant messaging tools and e-mails to establish a communication channel in all phases of the study. Teleconferences were also held to share knowledge among the research groups and to discuss the experimental procedure that the participants had to follow.

\section{Results}

In this section, we present the data analysis following the procedure presented above: the presentation of the descriptive statistics, the test of the hypotheses related to the main factor (Origin), the analysis of the influence of the cofactor Ability and the analysis of the post-experiment survey.

Please bear in mind that in some cases the groups are not balanced (there are more subjects in one group than in the other) owing to the fact that some subjects abandoned the experiment during its execution. 


\subsection{Descriptive statistics and exploratory analysis}

\begin{tabular}{|c|c|c|c|c|c|c|c|c|}
\hline \multirow[t]{3}{*}{ Origin } & \multicolumn{8}{|c|}{ MEffic } \\
\hline & \multicolumn{4}{|c|}{$\mathrm{RE}$} & \multicolumn{4}{|l|}{ FD } \\
\hline & $N$ & $\bar{X}$ & Median & SD & $N$ & $\bar{X}$ & Median & SD \\
\hline E-US1 & 20 & 0.00270 & 0.00283 & 0.00079 & 20 & 0.00273 & 0.00303 & 0.00072 \\
\hline R-US2 & 22 & 0.00261 & 0.00245 & 0.00106 & 28 & 0.00350 & 0.00352 & 0.00147 \\
\hline R-UB & 39 & 0.00217 & 0.00204 & 0.00087 & 39 & 0.00255 & 0.00183 & 0.00406 \\
\hline
\end{tabular}

Tables 6 and 7 show the descriptive statistics of the Maintainability measures, i.e., MEffec and MEffic respectively, grouped by the Origin of the UML diagrams. These tables contain the following data: number of subjects $(N)$, mean $(\bar{X})$, median, and standard deviation (SD).

At a glance, we can observe that when the subjects used FD diagrams they obtained better values (although the difference is very low in both measures when comparing means (only in the case of MEffec in R-UB are the results are better with RE diagrams). This indicates that there is a slight tendency towards FD diagrams improving the performance of software code maintainers.

\subsection{Influence of origin of diagram}

In order to test the formulated hypotheses $\left(\mathrm{H}_{1,0}, \mathrm{H}_{2,0}\right)$ we analyzed the effect of the main factor (i.e. Origin) on the measures considered (i.e., MEffec and MEffic) using the non- parametric Mann-Whitney or ANOVA test, depending on the normality of data (as explained in Section 3.7).

In the following subsections, the results for each measure of the Mann-Whitney U tests or ANOVA tests are shown in tables (Tables 8 and 9), in which the Origin column describes the independent variable, $p$-value is the statistical significance obtained, op is the estimated observed power of the test, es is the effect size, and $R$ describes whether the data obtained allows us to reject the null hypothesis, while the tendency of the data in the case of the null hypothesis being rejected is shown in the "in favour of. .." column.

The results obtained for each hypothesis will be commented on in their corresponding subsections.

For each measure, we first decided to analyze the data related to maintenance in general, as is presented in the formulated hypothesis. We then made the decision to analyze the results by dividing them by the type of maintenance, since there may have been differences between the results from the adaptive and the corrective maintenance.

\subsubsection{Testing maintenance effectiveness: $\operatorname{MEffec}\left(H_{1,0}\right)$}

The first row of Table 8 shows that we cannot reject $\mathrm{H}_{1,0}$ in the first experiment (E-US1) and the first replication (R-US2) given that their $p$-value is greater than 0.05 . Hence, here the different origins of UML diagrams had no effect on the subjectś effectiveness when performing the source code maintenance tasks. The observed power of the test is low, probably because of a small effect size, so we assume a $0.946(1-0.054)$ and a $0.755(1-0.245)$ estimated probability of a Type II error in our assertions. Given the low value of the observed power, we cannot obtain strong conclusions from E-US1 and R-US2.

In the case of the second replication, the $p$-value is 0.047 , i.e., lower than 0.05 , and we can therefore reject the null hypothesis. In this case we can assume that there is a difference in the maintenance effectiveness when using RE diagrams or FD diagrams in favor of RE diagrams. What is more, the power of the test is quite high in this case but it is still not sufficient for us to be confident of the results, because there is a probability of $65 \%$ of committing a Type II error in our assertion.

We also performed an analysis of the influence of the Origin on maintenance effectiveness per type of maintenance, i.e., adaptive and corrective maintenance. The results were not significant in all the cases because the $p$-value is always greater than 0.05 . The same occurred when the MEffec as regards the time spent maintaining the system (without relating this to the number of correct answers) was tested.

\subsubsection{Testing maintenance efficiency: $\operatorname{MEffic}\left(\mathrm{H}_{2,0}\right)$}

The numbers in Table 9 shows that in the case of E-US1 and RUB there are no significant effects (the $p$-values are not smaller than 0.05 ) as regards the Origin of UML diagrams on maintenance efficiency and that, in this case, the statistical power is still very low. But, if the null hypothesis were to be accepted, we would be assuming a 0.949 (i.e., 1-0.051) estimated probability of a Type II error.

But as was the case with MEffec, there is one case in which there is an influence of the Origin on the maintainability efficiency in favor of FD diagrams. In this case, in R-US2 the $p$-value is 0.049 , and the null hypothesis is therefore rejected.

Once again, an analysis of the influence of the Origin on maintenance efficiency per type of maintenance, i.e., adaptive and corrective maintenance, was performed. In this case, the results were not significant either (the $p$-values are greater than 0.05 ).

We also attempted to measure MEffic as regards the time spent maintaining the system, without relating this to the number of correct answers (as was done before). In this case, the $p$-value was again higher than 0.05 (i.e., $p$-value $=0.725$ ) but with a higher statistical power (i.e., op $=0.5$ ). The same result was obtained in the test with the MEffic measure as regards the time spent maintaining the system (without relating this to the number of correct answers).

\subsubsection{Integrating the obtained results through meta-analysis}

When the different effect sizes of the experiments need to be combined to obtain a global effect of a factor, the statistical technique used is that of meta-analysis. Although we have found some significant results: in favor of RE diagrams in relation to MEffec in

\section{Table 6}

Descriptive statistics for MEffec.

\begin{tabular}{|c|c|c|c|c|c|c|c|c|}
\hline \multirow[t]{3}{*}{ Origin } & \multicolumn{8}{|c|}{ MEffec } \\
\hline & \multicolumn{4}{|l|}{ RE } & \multicolumn{4}{|l|}{ FD } \\
\hline & $N$ & $\bar{X}$ & Median & SD & $N$ & $\bar{X}$ & Median & SD \\
\hline E-US1 & 20 & 0.641 & 0.6818 & 0.165 & 20 & 0.650 & 0.6818 & 0.148 \\
\hline R-US2 & 23 & 0.597 & 0.5909 & 0.223 & 28 & 0.667 & 0.6818 & 0.174 \\
\hline R-UB & 39 & 0.512 & 0.5000 & 0.171 & 39 & 0.441 & 0.4091 & 0.219 \\
\hline
\end{tabular}


Table 7

Descriptive statistics for MEffic.

\begin{tabular}{|c|c|c|c|c|c|c|c|c|}
\hline \multirow[t]{3}{*}{ Origin } & \multicolumn{8}{|c|}{ MEffic } \\
\hline & \multicolumn{4}{|l|}{$\mathrm{RE}$} & \multicolumn{4}{|l|}{ FD } \\
\hline & $N$ & $\bar{X}$ & Median & SD & $N$ & $\bar{X}$ & Median & SD \\
\hline E-US1 & 20 & 0.00270 & 0.00283 & 0.00079 & 20 & 0.00273 & 0.00303 & 0.00072 \\
\hline R-US2 & 22 & 0.00261 & 0.00245 & 0.00106 & 28 & 0.00350 & 0.00352 & 0.00147 \\
\hline R-UB & 39 & 0.00217 & 0.00204 & 0.00087 & 39 & 0.00255 & 0.00183 & 0.00406 \\
\hline
\end{tabular}

Table 8

Statistical relation between Origin of diagram (RE/FD) and Maintainability Effectiveness (MEffec).

\begin{tabular}{lllllll}
\hline \multirow{2}{*}{ Origin } & MEffec & & & & & \\
\cline { 2 - 7 } & Test & $p$-Value & op & es & $R$ & In favour of... \\
\hline E-US1 & Man-Withney & 0.957 & 0.054 & 0.001 & No & - \\
R-US2 & ANOVA & 0.202 & 0.245 & 0.033 & No & - \\
R-UB & Man-Withney & $\mathbf{0 . 0 4 7}$ & 0.352 & 0.033 & Yes & RE diagrams \\
\hline
\end{tabular}

Table 9

Statistical relation between Origin of diagram (RE/FD) and Maintainability Efficiency (MEffec).

\begin{tabular}{lllllll}
\hline \multirow{2}{*}{ Origin } & MEffic & & & & & \\
\cline { 2 - 7 } & Test & $p$-Value & op & es & $\mathrm{R}$ & In favour of... \\
\hline E-US1 & Man-Withney & 0.534 & 0.051 & 0.0003 & No & - \\
R-US2 & Man-Withney & $\mathbf{0 . 0 4 9}$ & 0.343 & 0.049 & Yes & FD diagrams \\
R-UB & Man-Withney & 0.272 & 0.088 & 0.004 & No & - \\
\hline
\end{tabular}

R-UB, and in favor of FD diagrams in relation to MEffic in R-US2, there are no significant results in the remaining cases. We have therefore decided to integrate the results of the different studies through a meta-analysis (in which the factor is the Origin of UML diagrams and how this affects the modifiability of source code), in order to explore if stronger results can be found. This technique has been used for the same purpose in other families of experiments, such as that shown in [43].

For each dependent variable, we computed the mean value obtained by the participants when using RE diagrams, minus the mean value they obtained with FD diagrams, and these values were then used to compute the Hedges' g metric [44], [45]. The overall conclusion was obtained by calculating the $\mathrm{Z}$ score based on the mean and standard deviation of the Hedges' $g$ statistics of the experiments. The global effect size was therefore obtained by using the Hedges' g metric, with the weights proportional to the experiment size:

$\bar{Z}=\frac{\sum_{i} w_{i} z_{i}}{\sum_{i} w_{i}}$

where $w_{i}=1 /\left(n_{i}-3\right)$ and $n_{i}$ is the sample size of the $i$-th experiment. The higher the value of Hedges' $g$, the higher the corresponding mean difference. An effect size of 0.5 indicates that the mean value obtained when using FD diagrams is half a standard deviation larger than the mean value obtained when not using them.

As suggested in [45], the effect size can be classified as: small (S) for values between 0 and 0.37 , medium (M) for values between 0.38 and 1.0 , and large $(\mathrm{L})$ for values above 1.00 .

The meta-analysis was performed by using the Comprehensive Meta-Analysis v2 tool [46]. For each measure, the tool produced the forest plots depicted in Figs. 5 and 6. The squares and diamonds are mostly proportional in size to each study's weight under the fixed effect model (see the 'Relative weight' column). The squares show the individual effect size of each experiment and the diamond shows the global effect size. The values of the Hedges' g metric are also reported. Positive values indicate that the use of FD diagrams improves the modifiability of source code. Negative values signify that RE diagrams are the best treatment.

If we focus on the results obtained for the MEffec variable (see Fig. 5), the total effect is in favor of using FD diagrams, but the global effect size obtained is not statistically significant since the $p$-value is greater than 0.05 . The value obtained for the Hedge's $g$ metric, i.e., 0.282, indicates a small size for the global effect. Similar results were obtained for MEffic (see Fig. 6), where the total effect is again in favor of FD diagrams, but the size of the effect is small. In both cases, if the coincidence interval were to be increased to $90 \%$ instead of $95 \%$, then the meta-analysis would be significant because the $p$-values would not be greater than 0.10 .

\subsection{Influence of Ability}

We tested whether the subjects' Ability influenced the results but. The effect of this factor could not be confirmed because the $p$-value found was higher than 0.05 in all of the cases (see Table 10), i.e., the subjects' Ability had no statistical influence on the results. This was expected owing to the balanced design of the experiment.

The interaction plot shown in Fig. 7 indicates that there was no interaction between Origin and Ability for MEffec. In the case of E-US1 and R-US2, high ability participants achieved better scores than low ability ones when both of them were using RE and FD diagrams. The interaction plot also suggests that the results achieved with FD diagrams are better than those obtained with RE diagrams for both high and low ability participants. This might be owing to the fact that RE diagrams contain too many details when compared with FD diagrams. In particular, RE sequence diagrams are twice as large in terms of messages when compared to FD diagrams. This could be because FD diagrams only contain logical messages between objects, and that messages between other kinds of objects are obviated, such as objects from Java packages, which are shown in RE diagrams. This difference between RE and FD diagrams is based on their nature, owing to the fact that human based diagrams contain less technical details than RE diagrams. In the case of R-UB, the effect of the combination of the Ability and the Origin is contrary to the other cases, i.e., the results achieved with RE diagrams are better than those obtained with FD diagrams for both high and low ability participants.

As with MEffec, the influence of the subjects' Ability and the Origin in the case of MEffic was also represented on an interaction plot. The interaction plot shown in Fig. 8 indicates that there was a clear interaction between Origin and Ability for MEffic. In this case, high Ability participants achieved better scores using the FD diagrams, and low Ability participants did better using the RE diagrams (except in the case of R-US2). This might be explained by the fact that RE diagrams have a very high traceability with source code, so inexperienced maintainers would prefer this kind of diagrams. However, experienced maintainers do not need very high traceability, because using FD diagrams might allow them to 
MEffec

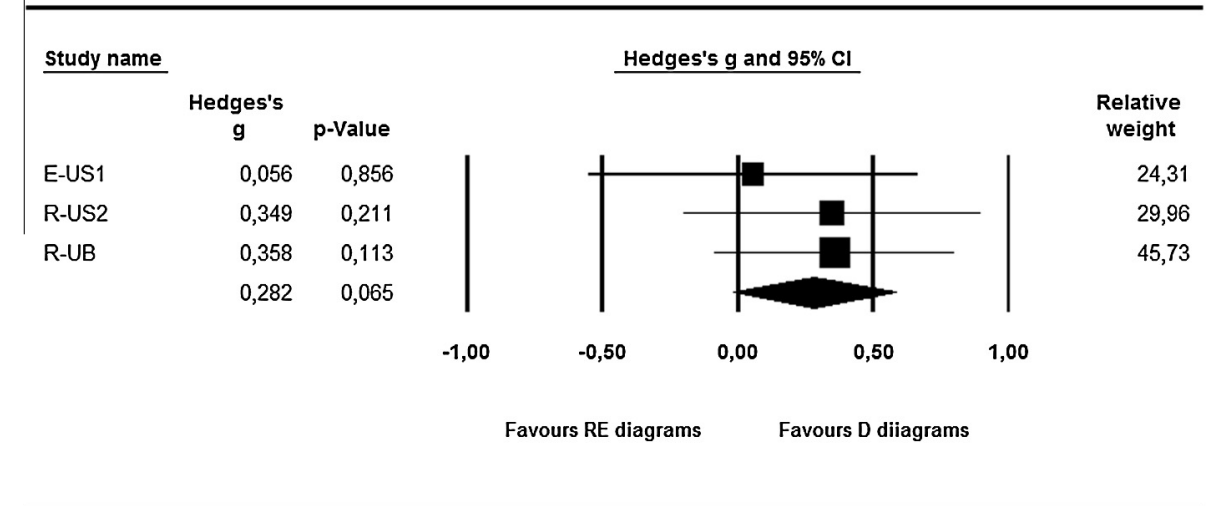

Fig. 5. Meta-analysis results for Maintainability Effectiveness (MEffec).

obtain sufficient information to attain a correct overview of how the system works.

\subsection{Post-experiment survey results}

The analysis of the answers to the post-experiment survey revealed that the time needed to carry out the modification tasks (Fig. 9) was not considered to be sufficient (more time was needed), and that the subjects considered that the performance of the tasks was of more or less low-medium difficulty (Fig. 10), independently of the particular treatment received. The need for more time to perform the tasks may have arisen from the fact that the measurement of the time needed was derived from the pilot study, which was performed by PhD students, who probably had higher ability and/or more experience than these Master's students, signifying that the less experienced subjects needed more time. We would also like to state that some subjects did not finish the questionnaire owing precisely to this lack of time.

We also asked about the subjects' perception of the adequacy of the LoD of the diagrams used. The majority of the subjects who received FD diagrams agreed with the LoD of the diagrams they received (i.e., they considered the LoD as the "right" amount of detail). In the case of those subjects who received RE diagrams, the majority agreed with the LoD presented, but of those subjects who did not, the majority required more LoD (Fig. 11).

The subjects who received FD diagrams experienced fewer difficulties when reading the diagrams used, in comparison with the RE group, as is shown in Fig. 12. We tested whether there was a difference as regards the difficulties experienced by subjects depending on the diagrams they used. This was done by using a Mann-Whitney test owing to the nature of the data, and this test was carried out by comparing the subjects' responses (from $1=$ very low to $5=$ very high) grouped by the UML diagrams that they had used (RE or FD diagrams). The results of the test show a significant difference ( $p$-value $=0.007$, which is lower than $\alpha=0.05)$. The power of the test is high (0.743), and this therefore allows us to state that the subjects who received RE diagrams experienced more difficulties when reading diagrams than those who received FD diagrams.

As part of the post-experiment survey, the subjects were required to indicate how useful the diagrams were for them in general as regards solving tasks. Class diagrams are considered useful in both groups, in more or less the same proportion (Fig. 13). Having said that, the majority of the subjects who received the RE diagrams commented that the sequence diagrams employed were not useful and were very difficult to understand, as opposed to the majority of the subjects in the D group who considered them helpful (Fig. 14). This finding may have been caused by the different complexities and varying LoD in the different kinds of diagrams, as explained in previous sections.

A Mann-Whitney test was then used (owing to the nature of the data) to test whether there was a difference as regards the subjects' perceived usefulness of the sequence diagrams depending on the diagrams they used. The test was carried out with the same configuration as explained in the test for the difficulties when reading the diagrams. The results of the test again show a significant difference ( $p$-value $=0.002$, which is lower than $\alpha=0.05$ ). The power of the test is high (0.88), and this therefore allows us to state that the subjects who received RE sequence diagrams considered them to be less helpful than the sequence diagrams in comparison to those who received FD sequence diagrams.

The results of the post-questions concerning the artifacts used to answer the questions led us to detect that almost all the subjects used the source code to solve the tasks. This was expected, in the sense that source code is needed when it is being maintained. The use of the source code was from $7 \%$ to $25 \%$ higher in the RE group than in the $\mathrm{D}$ group.

We then analyzed whether or not the subjects had used the diagrams (Table 11). Class diagrams were also used by the majority of subjects (except in the case of the D group in R-UB). These percentages are consistent with the subjective response provided in the post-experiment survey (see Fig. 13). In the case of the RE group, the subjects used class diagrams in more or less the same proportion for corrective or perfective tasks, but in the case of the D group, the subjects used from $7 \%$ to $27 \%$ more class diagrams for perfective tasks. This may have occurred because class diagrams provide the structure of the system, thus allowing maintainers to obtain an overview of the system faster, which would appear to be easier with the FD diagrams owing to their conciseness; this is more important for perfective tasks. If we focus on the use of sequence diagrams, we would like to highlight that their use was surprisingly low; in general, only $20-41 \%$ of the subjects used them. That is consistent with the RE group subjects' opinions (Fig. 14), in which they indicate that they did not use sequence diagrams, and they also believe that they are not useful diagrams for understanding the system during its maintenance. In the case of the $\mathrm{D}$ group, there is an inconsistency arising from the fact that the subjects did not use sequence diagrams in most of the tasks, even though they considered them to be useful (see Fig. 14). Subjects from both groups (except the RE group of R-US2) used the sequence diagram more in corrective tasks than in perfective tasks (a difference of $2-50 \%$ ). The reason for this could be that 


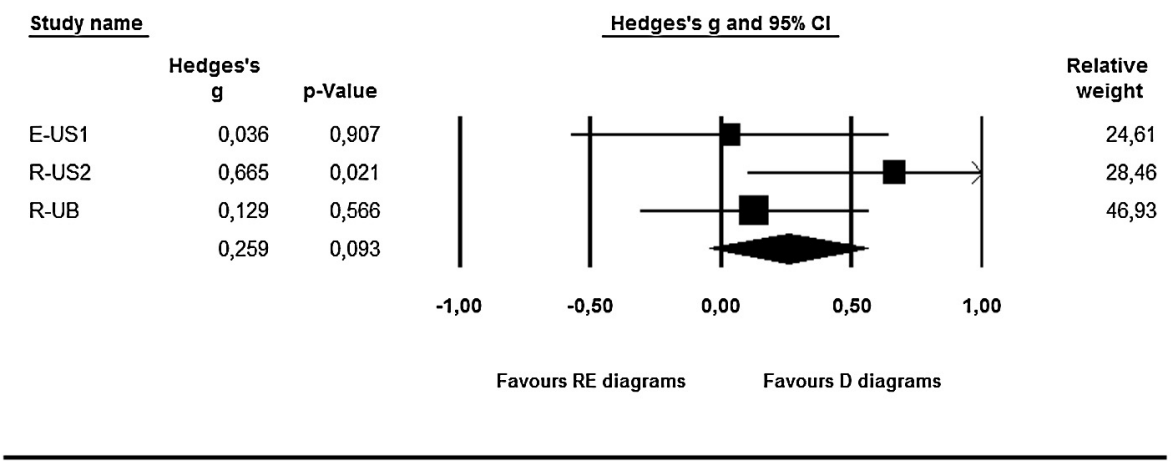

Fig. 6. Meta-analysis results for Maintainability Efficiency (MEffic).

Table 10

Statistical relation between ability and Maintainability Effectiveness (MEffec) and Efficiency (MEffic)

\begin{tabular}{lll}
\hline Ability & MEffec & MEffic \\
\hline E-US1 & 0.226 & 0.914 \\
R-US2 & 0.460 & 0.939 \\
R-UB & 0.470 & 0.995 \\
\hline
\end{tabular}

for corrective tasks, in which maintainers need to localize an error structure and behavior are needed, since the error might be caused by a structural error or by a behavior error.

\subsection{Summary and discussion of the data analysis}

The experiment (E-US1) was performed with 40 students from the University of Seville (Spain), and it was replicated in the same
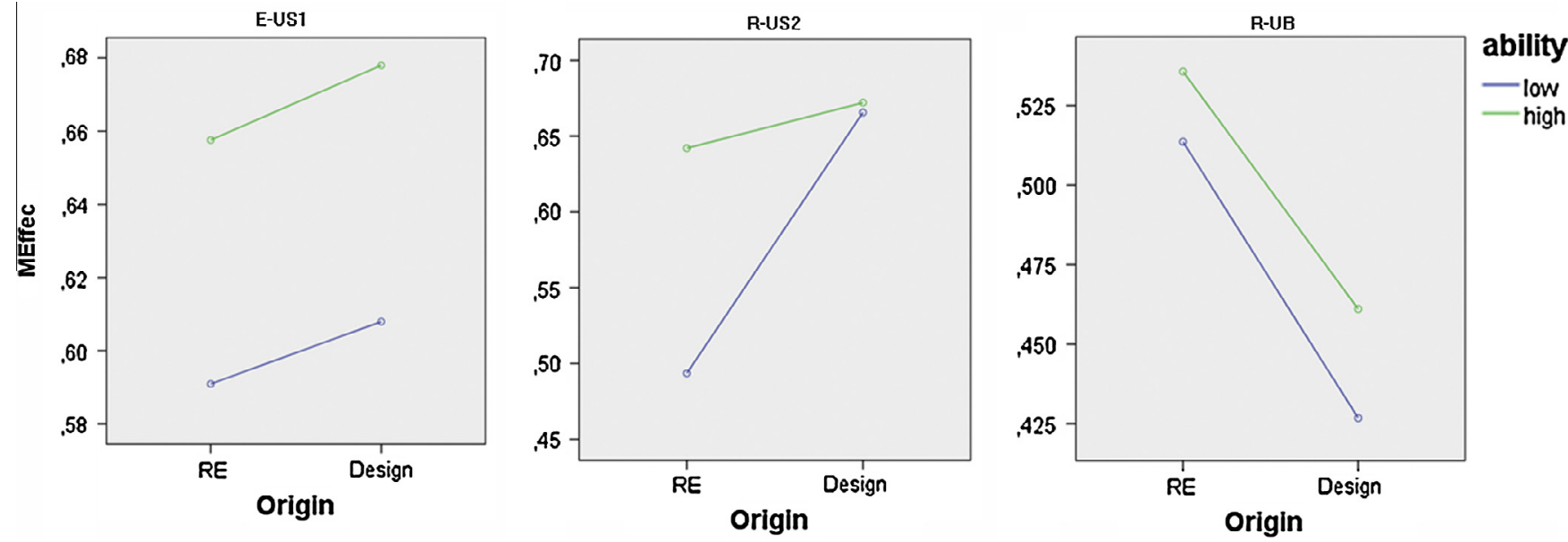

Fig. 7. Interaction between origin and ability for Maintainability Effectiveness (MEffec).
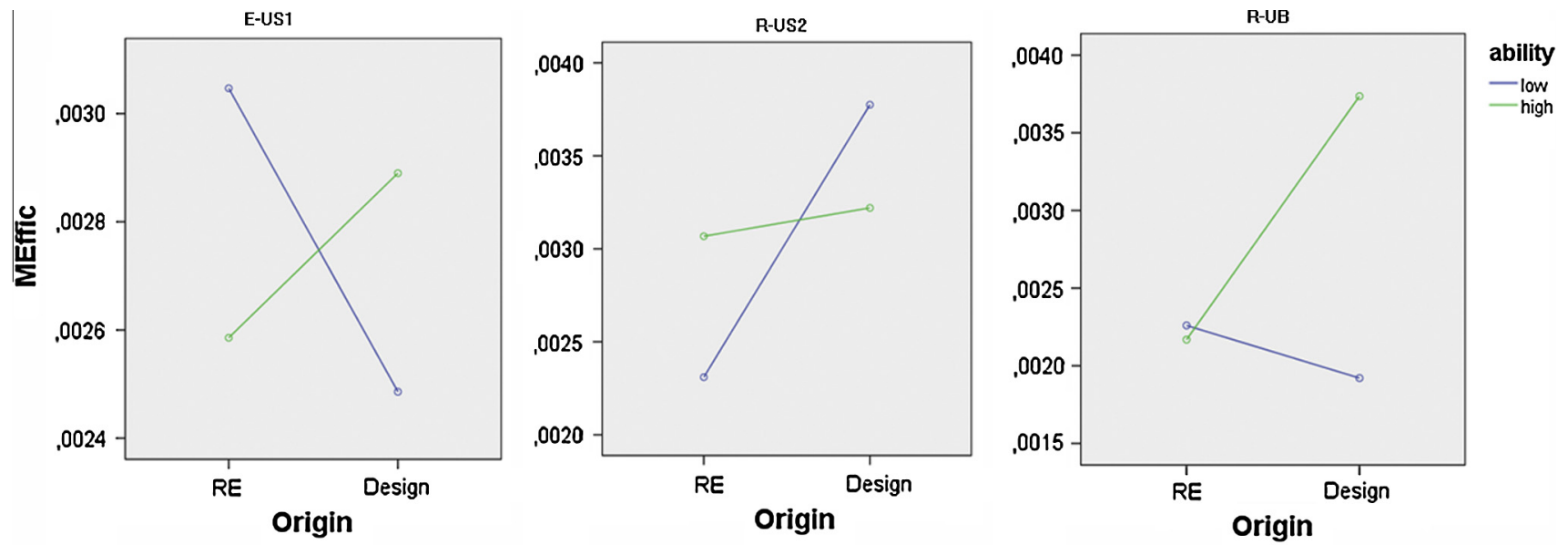

Fig. 8. Interaction between Origin and Ability for Maintainability Efficiency (MEffic). 


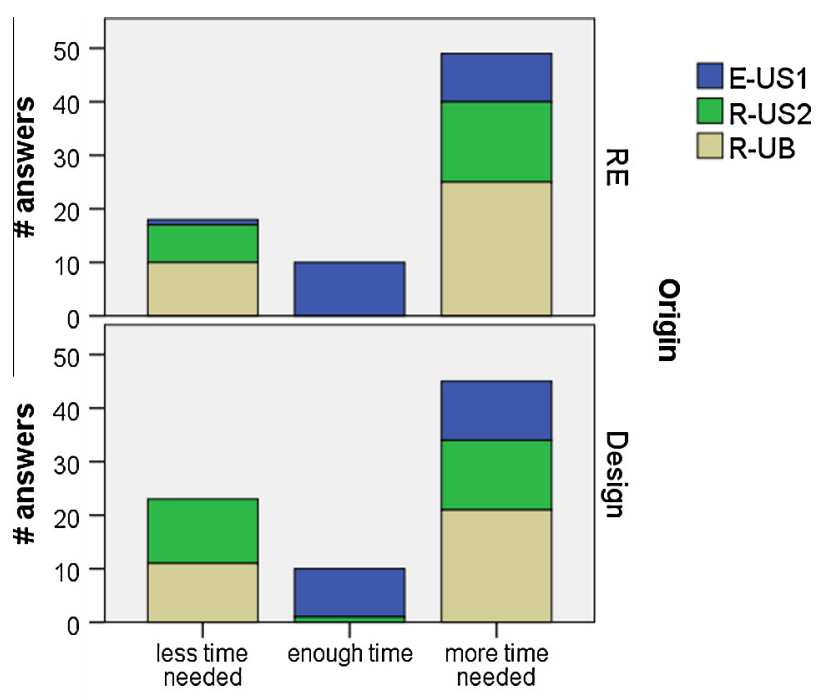

Adequacy of time

Fig. 9. Subjects' answers as regards adequacy of time provided.

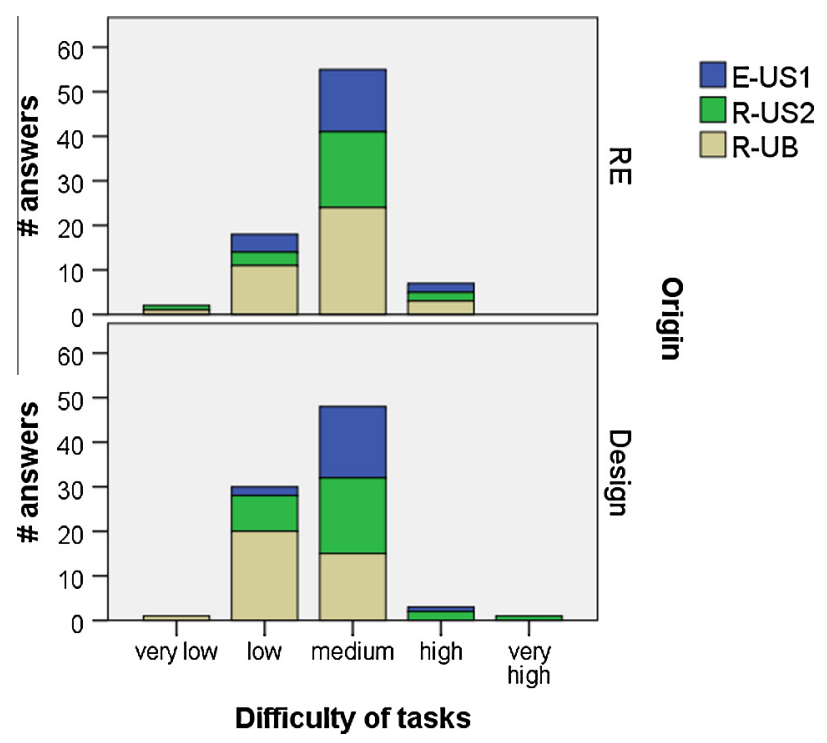

Fig. 10. Subjects' answers as regards difficulty of task.

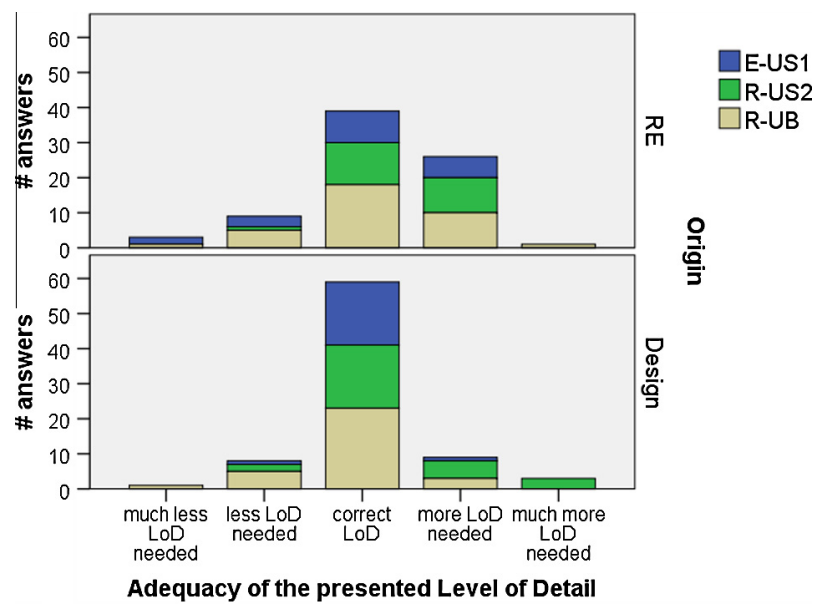

Fig. 11. Subjects' answers as regards adequacy of the LoD.

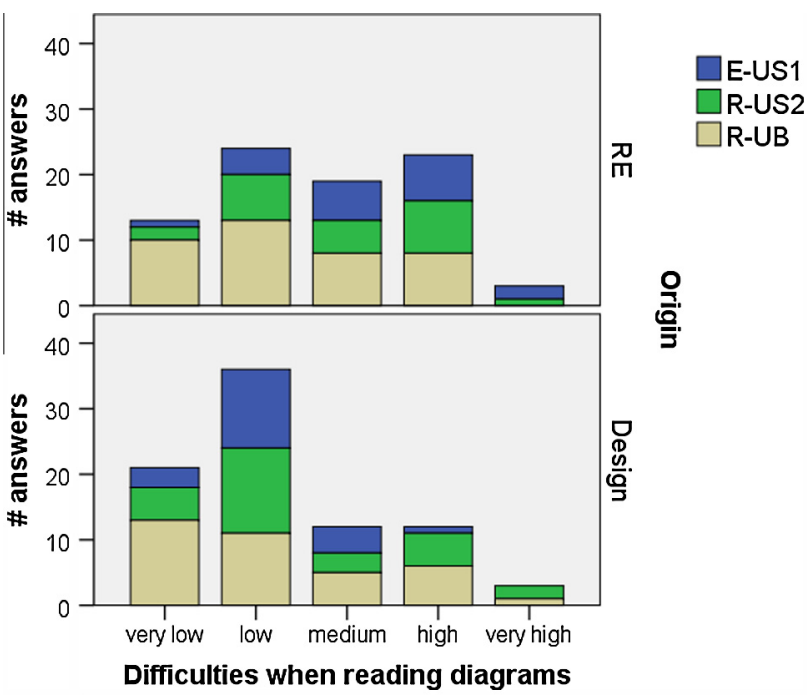

Fig. 12. Subjects' answers as regards to difficulties when reading diagrams.

university with 51 students (R-US2) and in the University of Bari, Italy, with 78 students (R-UB).

Descriptive statistic results show that subjects using FD UML diagrams obtained better values in both measures (except in the case of MEffec in R-UB), indicating that FD diagrams may, to some extent, improve the maintenance of the source code, but that the differences are very slight.

As regards the results of the statistical test, in almost all of the cases the variables (i.e., MEffec and MEffic) are not significantly affected by the Origin of the UML diagrams, i.e., the results of the tests performed did not allow us to reject any of the null hypotheses presented in section III in almost all cases, as all the significance levels are above 0.05 . The test powers are low, so the possibility of an error occurring as a result of accepting the null hypothesis is high. But if we focus on the case of R-US2, the results of the MEffic were influenced by the Origin, thus obtaining a high test power. The same occurred in the case of R-UB with MEffec, where there is a clear difference in favor of FD diagrams.

Despite the fact that the results were not conclusive in all the cases (which is not as positive as we expected), we have ensured that the experimental results were not influenced by other cofactors such as the subjects' Ability. If we focus on the interaction between Origin and Ability, in the case of E-US1 and R-US2, the subjects using FD diagrams achieved better results than those who used RE diagrams, in the case of both high and low ability participants. This may have been caused by the fact that RE diagrams contain too many details when compared with FD diagrams. In the case of R-UB, the effect of the combination of the Ability and the Origin is contrary to the other cases, i.e., the results achieved with RE diagrams are better than those obtained with FD diagrams in the case of both high and low ability participants. The low ability users obtained more benefits from RE diagrams than from FD diagrams in terms of efficiency owing to the high traceability between RE diagrams and code.

The results of the family of the experiments are summarized in Table 12.

After the individual analysis, a meta-analysis was performed in order to clarify the results. Its results also show a tendency in favor of FD diagrams for both measures (MEffec and MEffic). But in both cases, the results are not clearly evident owing to the values of the $p$-values.

Moreover, if we study the results of the post-experiment survey, we can see better subjective results for the FD diagrams. This 


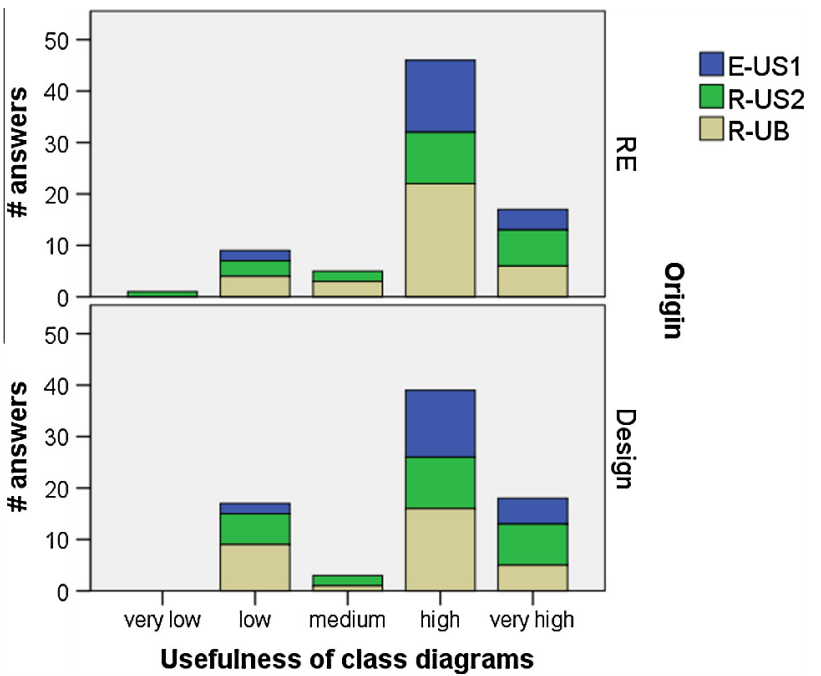

Fig. 13. Subjects' answers as regards usefulness of class diagram used.

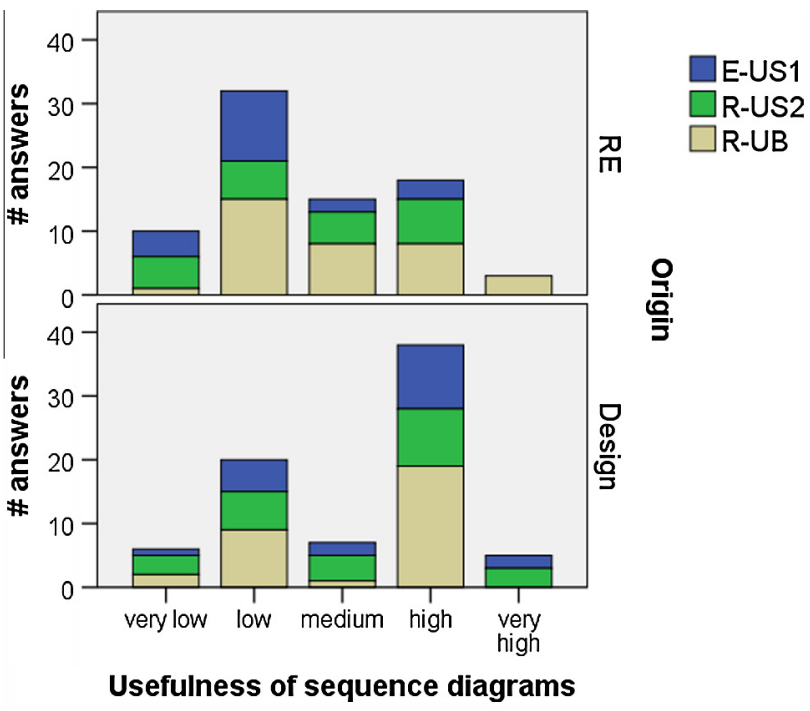

Fig. 14. Subjects' answers as regards usefulness of sequence diagrams used.

Table 11

Usage of UML diagrams for solving tasks.

\begin{tabular}{llllll}
\hline \multirow{2}{*}{ Exp. } & \multicolumn{2}{l}{ Usage of class diagrams } & & \multicolumn{2}{l}{ Usage of sequence diagrams } \\
\cline { 2 - 3 } & RE $(\%)$ & $D(\%)$ & & RE $(\%)$ & $D(\%)$ \\
\hline E-US1 & 80 & 74 & & 33 & 33 \\
R-US2 & 59 & 69 & & 23 & 29 \\
R-UB & 67 & 40 & & 41 & 20
\end{tabular}

is because the subjects who received RE diagrams did not believe their sequence diagrams to be useful, since they were not understandable. Significant results were obtained, showing that the subjects who received RE diagrams experienced more difficulties when reading the diagrams used; this is especially true as regards sequence diagrams. In the case of the D group, there was an inconsistency arising from the fact that the subjects did not use sequence diagrams in most of the tasks, even though they considered them to be useful.

We would like to underline that, according to the results of the post-question for each maintenance task concerning the use of artifacts in task solving, UML class diagrams were used in more or less in the same proportion as the source code for system understanding during the maintenance tasks. The sequence diagram was less widely-used, probably because of the nature of the tasks presented during the course of this experiment (more perfective maintenance tasks were required than corrective maintenance tasks). As stated previously, UML diagrams are not usually updated during maintenance tasks owing to the time constraints in realistic environments. But the high level of use of class diagrams during this experiment leads us to recommend companies to keep them up to date in order to improve maintainers' performances.

\section{Implications of the study}

No evident overall pattern emerges from the family of experiments of this study - especially when limiting to the statistics of the experiments: when considered individually 1 out of 3 experiments does not provide statistically significant results in favour of either RE or FD diagrams.

The experiments showed that high Ability participants achieved better scores using the FD diagrams, and low Ability participants did better using the RE diagrams (except in the case of R-US2). This might be explained by the fact that RE diagrams have a very high traceability with source code, so inexperienced maintainers would prefer this kind of diagrams. This group may find it difficult to make mentally bridge the gap between source code and high level class diagram. However, experienced maintainers do not need very high traceability, because using FD diagrams might allow them to obtain sufficient information to attain a correct overview of how the system works.

The key characteristics of RE and FD diagrams that we compare in this study are the following: RE diagrams are complete and close to source code. Man-made FD diagrams are almost never complete, but their strength seems to be the selective inclusion of information about the system and modest complexity of the diagrams.

Findings from the post-experiment survey show that subjects who received FD diagrams experienced fewer difficulties when reading the diagrams compared to the $\mathrm{RE}$ group ( $p$-value $=0.007$ ), especially with the class diagrams. At the same time, RE sequence diagrams were found less useful than FD sequence diagrams ( $p$-value $=0.002$ ). A possible explanation for this is that human engineers make (implicit) assessments regarding which information is important for understanding key aspects of the system and thus regarding which information should be included in a diagram. We believe the findings of our experiments indicate that FD class diagrams provide a more attractive balance between detail and relevant information than RE class diagrams. For sequence diagrams, it seems that current automated Reverse Engineering methods are not able to filter out the relevant information for maintenance tasks.

On the other hand, for performing the maintenance tasks in our study, participants found that source code source to be the most useful source of information, closely followed by the class diagrams. The participants therefore perceived that UML diagrams might add little value over access to the source code for the tasks in our study. This result is relevant for the researcher because it might be interesting to investigate the motivation guiding a software engineer when trusting in a source of information and how he/she exploits it to accomplish a maintenance task.

\section{Threats to validity}

We must consider certain issues which may have threatened the validity of the experiment [29]: 
Table 12

Summary of the results of the family of experiments.

\begin{tabular}{|c|c|c|c|c|c|c|}
\hline \multirow[t]{2}{*}{ Exp_ID } & \multicolumn{2}{|c|}{$\begin{array}{l}\text { Descriptive statistics (in } \\
\text { favour of...) }\end{array}$} & \multicolumn{2}{|c|}{$\begin{array}{l}\text { Influence of } \\
\text { origin }\end{array}$} & \multicolumn{2}{|c|}{$\begin{array}{l}\text { Influence of } \\
\text { ability }\end{array}$} \\
\hline & MEffec & MEffic & MEffec & MEffic & MEffec & MEffic \\
\hline E-US1 & FD & FD & $x$ & $x$ & $x$ & $x$ \\
\hline R-US2 & FD & FD & $x$ & $\checkmark$ & $x$ & $x$ \\
\hline R-UB & $\mathrm{RE}$ & FD & $\checkmark$ & $x$ & $x$ & $x$ \\
\hline
\end{tabular}

Legend.

Descriptive statistics:

"FD" = Better results when using FD diagrams than RE diagrams.

"RE" = Better results when using RE diagrams than FD diagrams.

“-” = No differences when using FD or RE diagrams.

Influence of origin:

$\boldsymbol{X}=$ Hypothesis not rejected $\rightarrow$ there is no significant difference in the results when working with FD or RE UML diagrams.

$\checkmark=$ Hypothesis rejected $\rightarrow$ there is a significant difference in the results when working with FD or RE UML diagrams (expected value).

Influence of ability:

$\boldsymbol{x}=$ Hypothesis not rejected $\rightarrow$ There is no significant difference in the results when working with high or low ability students (expected value).

$\checkmark=$ Hypothesis rejected $\rightarrow$ there is a significant difference in the results when working with high or low ability students.

- External validity: External validity can be threatened when experiments are performed with students, and the representativeness of these subjects may be doubtful in comparison to that of software professionals. In spite of this, the tasks to be performed did not require high levels of industrial experience, so we believe that this experiment could be considered appropriate, as it follows suggestions made in the relevant literature $[25,30,47]$. Working with students also implies various advantages, such as the fact that their prior knowledge is fairly homogeneous, there is the possible availability of a large number of subjects [48], and there is the opportunity to test experimental design and initial hypotheses [49]. An additional advantage of using novices as subjects in experiments on maintainability is that the cognitive complexity of the objects under study is not hidden by the subjects' experience. Nevertheless, it would be extremely interesting to carry out further replications of the experiment with practitioners.

Another threat to external validity concerns the experimental material used. The selected system is representative of a small industrial system. In addition, the experimental objects are small. The results of the experiments might be different when using bigger systems and experimental objects. The size of the experimental objects could also threaten the external validity of the results. The rationale for selecting the experimental objects used relies on the need (owing to time constraints) to simulate actual maintenance tasks related to small maintenance operations that novice software engineers and/or junior programmers may perform in a software company.

- Internal validity: Threats to internal validity were mitigated by the design of the experiment. Each subject was grouped by his/her results in the background questionnaire, so both groups had subjects with a similar skill level. In all the cases, the subjects' knowledge of UML and JAVA was reinforced by teaching them about UML diagrams and JAVA in a training session organized to take place the day before the experiment was carried out, but their knowledge was sufficient for them to understand the given system, and they all had roughly the same background (which was tested with a background questionnaire). Furthermore, all the participants found the material provided, the tasks, and the goals of the experiment to be clear, as the post-experiment survey results showed. Another safeguard was that the instrumentation was tested in a pilot study, to check its validity. In addition, mortality threats were mitigated by offering the subjects the possibility of performing similar tasks in the final exam of the course that they were taking. Another issue that is a potential threat is the exchange of information among the participants. We must emphasize that the participants were not allowed to communicate with each other, and were prevented from doing so by being monitored during the run of the experiment. When the experiment had concluded, the participants were asked to give back all the experimental material.

- Construct validity: This validity may be influenced by the measures used to obtain a quantitative evaluation of the subjects' performance, the maintenance tasks, and the post-experiment survey, in addition to social threats. We performed the experiment in a really short period of time owing to the subjects' constraints. The small amount of time that the students were given to perform the tasks could have influenced the results of this experiment, as could the small number of tasks, which was again owing to constraints on our subjects' time. The measures used were selected to achieve a balance between the correctness and completeness of the answers, which are well-known measures and are widely-used in this kind of experiments. The questionnaires were defined to obtain sufficiently complex questions, without them being too obvious. The post-experiment survey was designed using standard forms and scales. Social threats (e.g., evaluation apprehension) have been avoided, since the students were not graded on the results obtained. Absenteeism was avoided by performing similar tasks to the exercises that would appear in their final exam.

Based on the results of these pilot study, we consider that the two experimental objects fit the time constraints of the experiment in that they are sufficiently realistic for small maintenance operations that novice software engineers perform within a software company [50].

The experimental material delivered to the subjects consisted of the UML diagrams (class and sequence diagrams) and the JAVA source code of the system, the answer sheets, and the postexperiment survey.

- Conclusion validity: Conclusion validity concerns the data collection, the reliability of the measurement, and the validity of the statistical tests, all or any of which might affect the ability to draw a correct conclusion. Statistical tests were used to reject the null hypotheses, but the fact that subjects performed a small number of tasks provided us with few data points to work with. These particular statistical tests were selected by checking that they followed the specific assumptions related to their use. We have explicitly mentioned and discussed all those cases in which non-significant differences were present.

\section{Conclusions and future work}

As software maintenance takes up a major part of software projects, we are interested in investigating which documentation is more helpful in supporting maintainers when they have to maintain source code, This paper specifically presents a family of experiments that were carried out to investigate whether using either FD or RE UML diagrams (class and sequence diagrams) improve the maintainer's performance when modifying source code. We therefore wished to ascertain whether it is beneficial to build UML diagrams in the initial phases of development and kept them upto-date (which consumes more effort) or whether on the contrary it is better to rely on UML diagrams obtained by Reverse Engineering the source code.

The original experiment (E-US1) was carried out by 40 s-year students on a Master's Degree in Computer Science. The first replication (R-US2) was conducted by 51 students enrolled on the same degree and at the same university as the experiment. The second 
replication (R-UB) was performed with 78 s-year Laurea Degree students in Computer Science at the University of Bari.

The statistical results, and specifically the statistical test and the descriptive results, show a tendency towards obtaining better results when using UML diagrams (concretely class diagrams), that were hand-made during the design phase. Based on the qualitative results of the post-experiment survey, it is also important to note that the subjects preferred FD diagrams when understanding and maintaining a system. This is true even though their performance is not much better with FD diagrams in some cases, in comparison to their performance with RE diagrams. Because software maintenance is a human-based process, this highlighting of maintainers' perceptions in favor of using FD diagrams is very important.

In addition, we found that subjects who received RE diagrams experienced more difficulties when reading the diagrams, especially the sequence diagrams. Although the subjects who received FD diagrams felt that sequence diagrams were highly useful, as they expressed in the post-experiment survey, only a small number of subjects actually used the diagrams for the tasks in the experiment. In the case of the RE diagram group, the subjects did not use RE sequence diagrams. These subjects point out that these RE sequence diagrams are not very useful due to their low level of readability/high level of details. Even though the results of the family of experiments are not homogenous in all the experiments, the evidence shown in the MEffic test in R-US2, the results of the meta-analysis and the subjects' opinion extracted in the postquestionnaire survey point in the direction that software maintainers prefer to use FD UML diagrams over Reverse Engineered UML diagrams.

These results give us grounds to encourage software developers, albeit with caution, to follow a model-centric approach in projects with novel maintainers and small-sized systems related to wellknown domains. Nonetheless, other contexts should be explored in order to reaffirm the results in an industrial context by carrying out further replications with professionals, considering more complex systems related to non-well known domains.

The findings obtained imply beginning the development of a software system by creating UML diagrams, and in addition to keep them up-to-date, thereby making it easier to perform maintenance tasks. Class diagrams are important artifacts which are widely used and highly appreciated by maintainers. However, there is a doubt as to whether the documentation, and UML as part of it, will be maintained as the system evolves when a model-centric approach is used [51]. This goes against an effective use of the diagrams, a fact that suggests that we should recommend companies to keep their diagrams up-to-date and thus help their maintainers to perform the required tasks efficiently. The results obtained are therefore useful for all those companies that exploit this notation as a support for software maintainers when performing maintenance tasks.

The recommending on the use of Forward Designed UML diagrams (at least the class diagrams) assumes that UML diagrams were created during software development, and kept up-to-date during software maintenance. This requires an additional effort in the software lifecycle in comparison with the automated generation of UML diagrams from source code through Reverse Engineering techniques. Currently it seems that Reverse Engineering techniques are not able to abstract away less important information from the source code. Hence diagrams obtained via RE also require manual effort for simplifying them. The empirical investigation of whether this extra effort is worthwhile is still pending, but would provide us with knowledge regarding the return on investment of UML modeling in software maintenance. This topic has not been explored in detail, and this is necessary if we are to discover whether UML will have a widely acceptation in industry and how UML can gain a widely adoption in industry.

\section{Acknowledgments}

This research has been funded by the GEODAS-BC Project (Ministerio de Economía y Competitividad and Fondo Europeo de Desarrollo Regional FEDER, TIN2012-37493-C03-01). The authors would like to thank the students who have cooperated in the experiments.

\section{References}

[1] M. Abbes, F. Khomh, Y.-G. Gueheneuc, G. Antoniol, An empirical study of the impact of two antipatterns, blob and spaghetti code, on program comprehension, in: Presented at the Proceedings of the European Conference on Software Maintenance and Reengineering (CSMR'2011), 2011, pp. 181-190.

[2] S.C.B. de Souza, N. Anquetil, K.M. de Oliveira, A study of the documentation essential to software maintenance, in: Presented at the Proceedings of the International Conference on Design of communication: documenting \& designing for pervasive information (SIGDOC'2005), 2005, pp. 68-75.

[3] S. Tilley, S. Huang, A Qualitative Assessment of the Efficacy of UML Diagrams as a Form of Graphical Documentation in Aiding Program Understanding, in: Presented at the Proceedings of the 21st Annual International Conference on Systems Documentation (SIGDOC'2003), 2003, pp. 184-191.

[4] E. Tryggeseth, Report from an experiment: impact of documentation on maintenance, J. Empirical Softw. Eng. 2 (2) (1997) 201-207.

[5] OMG, The Unified Modeling Language. Documents associated with UML version 2.3, 2010, <http://www.omg.org/spec/UML/2.3>.

[6] A. Nugroho, M.R.V. Chaudron, Evaluating the impact of UML modeling on software quality: An industrial case study, in: Presented at the Proceedings of the 12th International Conference on Model Driven Engineering Languages and Systems (MODELS'09), vol. 181-195, 2009.

[7] A. Nugroho, B. Flaton, M.R.V. Chaudron, Empirical Analysis of the Relation between Level of Detail in UML Models and Defect Density, in: Presented at the Proceedings of the International Conference on Model Driven Engineering Languages and Systems (MoDELS'2008), 2008, pp. 600-614.

[8] E. Arisholm, L.C. Briand, S.E. Hove, Y Labiche, The impact of UML documentation on software maintenance: an experimental evaluation, IEEE Trans. Software Eng. 32 (6) (2006) 365-381.

[9] W.J. Dzidek, E. Arisholm, L.C. Briand, A realistic empirical evaluation of the costs and benefits of UML in software maintenance, IEEE Trans. Software Eng. 34 (3) (2008) 407-432.

[10] B. Berenbach, S. Konrad, Putting the 'Engineering' into Software Engineering with Models, in: Presented at the Proceedings of the International Workshop on Modeling in Software Engineering (MISE '07), ICSE Workshop, 2007, p. 4.

[11] B. Anda, K. Hansen, I. Gullesen, H.K. Thorsen, Experiences from introducing UML-based development in a large safety-critical project, Empirical Softw. Eng. 11 (4) (2006) 555-581. December.

[12] G. Scanniello, C. Gravino, M. Genero, J.A. Cruz-Lemus, G. Tortora, On the Impact of UML Analysis Models on Source Code Comprehensibility and Modifiability, Acm. T. Softw. Eng. Meth. 23 (2) (2013). Art.13.

[13] A. Nugroho, M.R.V. Chaudron, A Survey of the Practice of Design - Code Correspondence Amongst Professional Software Engineers, in: Proceedings of the First International Symposium on Empirical Software Engineering and Measurement (ESEM'2007), Washington, DC, USA, 2007, pp. 467-469.

[14] A. Nugroho, Level of detail in UML models and its impact on model comprehension: a controlled experiment, Inf. Softw. Technol. 51 (12) (2009) 1670-1685.

[15] A.M. Fernández-Sáez, M. Genero, M.R.V. Chaudron, Does the Level of Detail of UML Models Affect the Maintainability of Source Code?", in: Proceedings of the Experiences and Empirical Studies in Software Modelling Workshop (EESSMod'11) at MODELS 2011, Wellington, New Zealand, 2012, pp. 133147.

[16] R. Perez-Castillo, I.G.-R. de Guzman, M. Piattini, C. Ebert, Reengineering technologies, IEEE Softw. 28 (6) (2011) 13-17.

[17] H. Osman, D. Stikkolorum, A. van Zadelhoff, M.R.V. Chaudron, UML Class Diagram Simplification: What is in the developer's mind?", in: Presented at the Proceedings of the 2012th international conference on Models in Software Engineering (MODELS'2011), Experiences and Empirical Studies in Software Modelling Workshop (EESSMoD'2012), 2012.

[18] A.M. Fernández-Sáez, M.R.V. Chaudron, M. Genero, I. Ramos, Are forward designed or reverse-engineered UML diagrams more helpful for code maintenance?: a controlled experiment", in: Proceedings of the 17th International Conference on Evaluation and Assessment in Software Engineering, New York, NY, USA, 2013, pp. 60-71.

[19] A.M. Fernández-Sáez, M. Genero, M.R.V. Chaudron, Empirical studies concerning the maintenance of UML diagrams and their use in the maintenance of code: a systematic mapping study, Inf. Softw. Technol. 55 (7) (2013) 1119-1142.

[20] G. Scanniello, C. Gravino, G. Tortora, Does the Combined use of Class and Sequence Diagrams Improve the Source Code Comprehension? Results from a Controlled Experiment", in: Proceedings of the Experiences and Empirical Studies in Software Modelling Workshop (EESSMoD́2012), 2012.

[21] A. Karahasanovic, R. Thomas, Difficulties experienced by students in maintaining object-oriented Systems: an empirical study, in: Proceedings of 
the Australasian Computing Education Conference (ACE'2007), 2007, pp. 8187.

[22] G. Scanniello, C. Gravino, G. Tortora, Investigating the Role of UML in the Software Modeling and Maintenance - A Preliminary Industrial Survey, in: Proceedings of the 12th International Conference on Enterprise Information Systems (ICEIS'2010), Funchal, Madeira, Portugal, 2010, vol. 3, pp. 141-148.

[23] B. Dobing, J. Parsons, How UML is used?, Commun ACM 49 (5) (2006) 109-113.

[24] M. Genero, A.M. Fernández-Sáez, H.J. Nelson, G. Poels, M. Piattini, A systematic literature review on the quality of UML models, J. Database Manage. 22 (2011) 46-70. July.

[25] V. Basili, F. Shull, F. Lanubile, Building knowledge through families of experiments, IEEE Trans. Software Eng. 25 (4) (1999) 456-473.

[26] K.R. Popper, The Logic of Scientific Discovery, Hutchinson \& Co, Cambridge, 1959.

[27] N. Juristo, A. Moreno, Basics of Software Engineering Experimentation, Kluwer Academic Publishers, 2001.

[28] A. Jedlitschka, M. Ciolkowoski, D. Pfahl, Reporting experiments in software engineering, in: F. Shull, J. Singer, D.I.K. Sjøberg (Eds.), Guide to Advanced Empirical Software Engineering, Springer Verlag, 2008.

[29] C. Wohlin, P. Runeson, M. Höst, M.C. Ohlsson, B. Regnell, A. Wesslén, Experimentation in Software Engineering: an Introduction, Springer, Norwell, MA, USA, 2012.

[30] V. Basili, D. Weiss, A methodology for collecting valid software engineering data, IEEE Trans. Software Eng. 10 (6) (1984) 728-738.

[31] M. Grossman, J.E. Aronson, R.V. McCarthy, Does UML make the grade? Insights from the software development community, Inf. Softw. Technol. 47 (6) (2005) 383-397. April.

[32] J. Erickson, K. Siau, Theoretical and practical complexity of modeling methods, Commun. ACM 50 (8) (2007) 46-51.

[33] W. Heijstek, M.R.V. Chaudron, Empirical Investigations of Model Size Complexity and Effort in a Large Scale, Distributed Model Driven Development Process, in: presented at the 35th Euromicro Conference on Software Engineering and Advanced Applications, 2009. SEAA '09, 2009, pp. 113-120.

[34] R.E. Kirk, Experimental Design. Procedures for the Behavioural Sciences, Brooks/Cole Publishing Company, 1995.

[35] ISO/IEC, ISO/IEC 14764-1999: Software Engineering Maintenance, 1999.

[36] E.B. Swanson, The dimensions of maintenance, in: Proceedings of the 2nd International Conference on Software Engineering (ICSE 1976), San Francisco, California, United States, 1976, pp. 492-497.
[37] A.N. Oppenheim, Questionnaire Design, Interviewing and Attitude Measurement, Pinter Publishers, 1992.

[38] D. Sheskin, Handbook of Parametric and Nonparametric Statistical Procedures, Chapman and Hall, Fourth, 2007.

[39] J.L. Devore, N. Farnum, Applied Statistics for Engineers and Scientists. Duxbury, 1999.

[40] SPSS, SPSS 12.0, Syntax Reference Guide. Chicago, USA: SPSS Inc., 2003.

[41] F. Shull, M.G. Mendonça, V.R. Basili, J. Carver, J.C. Maldonaldo, S. Fabbri, G.H. Travassos, M.C. Ferreira, Knowledge-sharing issues in experimental software engineering, Empirical Softw. Eng. 9 (1-2) (2004) 111-137.

[42] S. Vegas, N. Juristo, A. Moreno, M. Solari, P. Letelier, Analysis of the influence of communication between researchers on experiment replication, in: Proceedings of the ACM/IEEE international Symposium on Empirical Software Engineering (ISESE'2006), 2006, pp. 28-37.

[43] J.A. Cruz-Lemus, M. Genero, D. Caivano, S. Abrahão, E. Insfrán, J.A. Carsí, Assessing the influence of stereotypes on the comprehension of UML sequence diagrams: a family of experiments, Inf. Softw. Technol. 53 (12) (2010) 13911403.

[44] L.V. Hedges, I. Olkin, Statistical Methods for Meta-Analysis, Academia Press, 1985.

[45] V. Kampenes, T. Dybå, J.E. Hannay, D.I.K. Sjoberg, A systematic review of effect size in software engineering experiments, Inf. Softw. Technol. 49 (11-12) (2007) 1073-1086.

[46] Biostat, Comprehensive Meta-Analysis v2. Englewood, NJ, USA: Biostat, 2006.

[47] M. Höst, B. Regnell, C. Wohlin, Using students as subjects - a comparative study of students and professionals in lead-time impact assessment, J. Empirical Softw. Eng. 5 (2000) 201-214.

[48] J. Verelst, The influence of the level of abstraction on the evolvability of conceptual models of information systems, in: Proceedings of the International Symposium on Empirical Software Engineering (ISESE'04), 2004, pp. 17-26.

[49] D.I.K. Sjøberg, J.E. Hannay, O. Hansen, V.B. Kampenes, A. Karahasanovic, N. Liborg, A.C. Rekdal, A survey of controlled experiments in software engineering, IEEE Transact. Softw. Eng. 31 (9) (2005) 733-753.

[50] D. Cohen, M. Lindvall, P. Costa, An introduction to agile methods, Adv. Comput. 62 (2004) 2-67.

[51] M. Petre, UML in practice, in: Proceedings of the 2013 International Conference on Software Engineering (ICSE'2013), San Francisco, CA, USA, 2013, pp. 722731. 